\title{
Quantization of Prior Probabilities for Collaborative Distributed Hypothesis Testing
}

\author{
Joong Bum Rhim, Student Member, IEEE, Lav R. Varshney, Member, IEEE, and \\ Vivek K Goyal, Senior Member, IEEE
}

\begin{abstract}
This paper studies the quantization of prior probabilities, drawn from an ensemble, in distributed detection with data fusion by combination of binary local decisions. Design and performance equivalences between a team of $N$ agents and a more powerful single agent are obtained. Effects of identical quantization and diverse quantization on mean Bayes risk are compared. It is shown that when agents using diverse quantizers interact to agree on a perceived common risk, the effective number quantization levels is increased. With this collaboration, optimal diverse regular quantization with $K$ cells per quantizer performs as well as optimal identical quantization with $N(K-1)+1$ cells per quantizer. Similar results are obtained for the maximum Bayes risk error criterion.
\end{abstract}

Index Terms-Bayesian hypothesis testing, Bregman divergence, mean Bayes risk minimization, quantization theory, team theory.

\section{INTRODUCTION}

C ONSIDER a team of $N$ agents that aims to collaboratively choose between hypotheses $h_{0}$ and $h_{1}$ after each agent obtains a noisy observation. Local decision making proceeds in parallel, with all agents synchronously obtaining private observations, making hard decisions locally, and sending decisions to the fusion center, without any knowledge of other agents' decisions. The fusion center has some fixed fusion rule known to all agents that generates a global decision based only on local decisions. Fusion rules that are deterministic and symmetric are of the $L$-out-of- $N$ form whereby the global choice is $h_{1}$ when $L$ or more agents choose $h_{1}$. Examples are the MAJORITY rule $\left(L=\left\lceil\frac{N+1}{2}\right\rceil\right)$ and the OR rule $(L=1)$. With known prior probabilities for the object state, the local decision rules and the fusion rule can be analyzed and optimized as standard Bayesian hypothesis testing.

Symmetric combining of binary decisions arises often in human affairs. Decision making by juries or committees has been analyzed in economics and political science to understand rational decision rules when the human decision makers possess

Manuscript received September 12, 2011; revised January 10, 2012 and April 24, 2012; accepted May 03, 2012. Date of publication May 22, 2012; date of current version August 07, 2012. The associate editor coordinating the review of this manuscript and approving it for publication was Prof. Joseph Tabrikian. This work was supported by the National Science Foundation by Grants 0729069 and 1101147 and the Korea Foundation for Advanced Studies (KFAS) Fellowship. The material in this paper was presented in part at the IEEE Data Compression Conference, Snowbird, UT, March 2011.

J. B. Rhim and V. K Goyal are with the Department of Electrical Engineering and Computer Science and the Research Laboratory of Electronics, Massachusetts Institute of Technology, Cambridge, MA 02139 USA (e-mail: jbrhim@mit. edu; vgoyal@mit.edu.

L. R. Varshney is with the IBM Thomas J. Watson Research Center (e-mail: varshney@alum.mit.edu).

Digital Object Identifier 10.1109/TSP.2012.2200890 a common preference for two alternatives [1]. A long-running thread in economics treats human agents as Bayesian decision makers [2], and this is supported by psychological research [3] as well. A key human limitation is a prime motivation for this work: Human decision makers are known to think categorically - first assigning a decision-making problem to a category and then applying a rule appropriate for that category - due to their limited information processing capacities [4]. The quality of decisions depends on the individuals' categorization schemes. Our tools and techniques are all from signal processing, and there is increasing interest among signal processing researchers to study the social world [5].

Here, we consider an ensemble of objects, generally uncountably many. Each agent is only able to apply a decision rule that depends on a category of the object. We limit our attention to binary hypothesis testing, so a prior distribution is specified by a single scalar

$$
p_{0}=\mathbb{P}\left(H=h_{0}\right)=1-\mathbb{P}\left(H=h_{1}\right) .
$$

We model categories as a partition of the possible values for $p_{0}$ into single intervals. From an agent's perspective, any object belongs to one of $K$ categories

$$
\begin{aligned}
\mathcal{C}_{1} & =\left\{\text { objects with } p_{0} \in\left[0, b_{1}\right)\right\} \\
\mathcal{C}_{2} & =\left\{\text { objects with } p_{0} \in\left[b_{1}, b_{2}\right)\right\} \\
\vdots & \\
\mathcal{C}_{K} & =\left\{\text { objects with } p_{0} \in\left[b_{K-1}, 1\right]\right\} .
\end{aligned}
$$

The agent treats any object in $\mathcal{C}_{k}$ as having prior probability $a_{k}$ rather than the true prior probability $p_{0}$. Due to categorization, the agent only needs $K$ decision rules - one for each category - no matter how many objects are there. In contrast, if the agent performs Bayesian hypothesis testing with exact prior probabilities, then the agent needs to have as many decision rules as objects.

In this model, categorization is a mapping from the true prior probability space $[0,1]$ to one of the $K$ discriminable values $\left\{a_{1}, \ldots, a_{K}\right\}$, which is a form of regular scalar quantization. Quantization performance is measured by the quality degradation of decisions made based on the quantized prior probability; this is a Bregman divergence called Bayes risk error [6]. We model $p_{0}$ as a realization of a random variable $P_{0}$ with probability density function $f_{P_{0}}\left(p_{0}\right)$, and averaging the Bayes risk error over $P_{0}$ gives mean Bayes risk error (MBRE). Except in Section VI, our goal is to optimize performance with respect to MBRE, with $f_{P_{0}}$ given. We consider two cases: when all agents use identical quantizers and when they use different quantizers. 
The traditional advantage of having multiple agents in hypothesis testing problems is obtaining more observations about objects: agents observe different noise realizations and reduce the effects of noise by information aggregation. New here, we show that diversity among agents' mapping schemes for prior probabilities can decrease the chance that inexact prior probabilities lead to wrong decisions. Diversity potentially helps each agent in cancelling others' wrong decisions so that the quality of a global decision can be improved on average.

It is assumed in this paper that as part of their collaboration, agents share their quantized prior probabilities with each other. We introduce a new criterion for decision making - perceived common risk - which is computed from the shared values and used by all the agents. Under this collaboration, optimal design of the prior-probability quantizers enables $N$ agents with different $K$-level regular quantizers to perform as well as $N$ agents with identical $(N(K-1)+1)$-level quantizers.

The precise study of quantization of prior probabilities in Bayesian hypothesis testing was recently initiated in [7], which focuses on the minimum MBRE quantizer of a single agent. Quantization of prior probabilities to minimize maximum Bayes risk is considered in [8]. The study of quantization of prior probabilities in distributed hypothesis testing by three agents appears in [9], work generalized herein. We use a single set of Bayes costs as an element of making the agents a team in the sense of Marschak and Radner [10], i.e., having a common goal. An alternative is for each agent to have potentially-different Bayes costs. This introduces game-theoretic considerations as described in [11].

Most previous work on the effect of quantization in Bayesian distributed detection is focused on the quantization of observations [12]-[14] or the communication topology and rates among agents [15] and/or to the fusion center [16], [17]. We do not consider quantization of observations here, though it may be noted that quantization outside of the system designer's control could be incorporated into the likelihood functions.

Section II provides additional background and motivation from human teams. The group decision-making model that we are considering is described in Section III. In Section IV, we discuss the effect of quantization of prior probabilities on decision making and compare performances of the teams of agents that use identical quantizers and that use different quantizers. Examples of optimal quantizers obtained from our design algorithm are presented in Section V. In addition to the mean Bayes risk error, the maximum Bayes risk error is considered in Section VI. Section VII concludes the paper.

\section{BACKGRound: Decisions By Human TeAms}

Although the mathematical results presented herein may be broadly applicable, our primary motivation is to introduce an analysis framework under which to study decision making by teams of human agents. These agents are restricted to a "categorical model of cognition," or more simply "categorical thinking" due to information-processing limitations well described in psychology and economics [4], [18]-[20]. This categorical thinking reduces the complexity of human decision making, and the human agents in this paper are assumed to know their limitations and optimize their strategy under the limitations, which is called "costly rationality" [21].

We look at teams of agents that work together to make decisions by voting [1] and one focus is on the benefit of diversity of categorization - cognitive diversity or diversity of training - among the agents.

Here is one particular example: a team of physicians that together make a binary decision, say on whether to implement a particular therapy. The key elements of a model of team decision making cast in this setting are as follows:

- An ensemble of problems is like a set of patients. One patient is to be treated. Let us say the patient collects votes and chooses to undergo the therapy if at least $L$ out of $N$ physicians recommend it.

- Categorization by prior probability is like forming risk groups (very high, high, medium, low, very low). Physicians may reasonably assign risk groups differently.

- A coordination stage of sharing quantized prior probabilities would involve the physicians sharing their opinions of the risk group of the patient.

- Each agent uses the quantized prior probability and a private observation, which is to say that a physician uses a risk group and a medical test within her expertise to make her recommendation. Human agents like physicians simply do not use something like a continuous-valued prior probability - they use categories. This is partly due to the aggregation into categories that is necessary for interpreting medical studies [22, Sec. 5.3].

- The fusion center collects votes from the agents; in our case, the patient collects the recommendations of the physicians. Of course, a patient could collect much more information, but this may place unreasonable burden on the patient - he needs to be able to rely on the physicians to distill information down to binary recommendations.

- From physicians' points of view, they treat many patients who have different prior probabilities. They may design their categories to minimize the overall population-wide risk due to wrong decisions.

- Our results indicate the benefit of diversity of quantization of prior probabilities, which means of the physicians using different risk group categorizations.

Although our results include various optimizations, we are not asserting that human agents actually perform these optimizations explicitly. By studying optimal performance under certain constraints, we are able to draw certain qualitative conclusions [21]. Though, it should be noted that human reasoning often approximates Bayes-optimal reasoning [2], [3], [23], [24].

\section{Distributed Detection and Data Fusion Model}

A binary hypothesis test for a given object is performed by a team of $N$ agents. The object is in state $H=h_{0}$ with probability $p_{0}$ and in state $H=h_{1}$ with probability $1-p_{0}$. The agents have a common goal to minimize the cost due to the global decision; the cost for false alarm (misjudgment of $h_{0}$ as $h_{1}$ ) is $c_{10}$ and the cost for missed detection (misjudgment of $h_{1}$ as $h_{0}$ ) is $c_{01}$ for all agents. For simplicity, we consider zero cost for correct decisions. 
The observation $Y_{i}$ by Agent $i$ is conditionally independent of $Y_{j}, j \neq i$, and governed by the likelihood function $f_{Y_{i} \mid H}\left(y_{i} \mid H=h_{m}\right)$. Each agent makes a hard local decision $\hat{H}_{i} \in\left\{h_{0}, h_{1}\right\}$ based on its observation and the prior probability it believes. The local decision is transferred to a fusion center to be merged with other agents' decisions by some fixed fusion rule. We consider symmetric fusion rules, which are described as $L$-out-of- $N$ rules for a specific $1 \leq L \leq N$. This fusion rule returns $\hat{H}=h_{1}$ if at least $L$ agents declare $h_{1}$; otherwise, it returns $\hat{H}=h_{0}$.

Each individual agent makes the two types of errors with the following conditional probabilities:

$$
\begin{aligned}
& P_{e, i}^{\mathrm{I}}=\mathbb{P}\left(\hat{H}_{i}=h_{1} \mid H=h_{0}\right) \\
& P_{e, i}^{\mathrm{II}}=\mathbb{P}\left(\hat{H}_{i}=h_{0} \mid H=h_{1}\right) .
\end{aligned}
$$

Then, from the $L$-out-of- $N$ fusion rule, the conditional probabilities of the global decision being in error are

$$
\begin{aligned}
& P_{E}^{\mathrm{I}}=\sum_{n=L}^{N} \sum_{\substack{I \subseteq[N] \\
|\bar{I}|=n}} \prod_{i \in I} P_{e, i}^{\mathrm{I}} \prod_{j \in[N] \backslash I}\left(1-P_{e, j}^{\mathrm{I}}\right) \\
& P_{E}^{\mathrm{II}}=\sum_{n=N-L+1}^{N} \sum_{\substack{I \subset[N] \\
|\subset|=n}} \prod_{i \in I} P_{e, i}^{\mathrm{II}} \prod_{j \in[N] \backslash I}\left(1-P_{e, j}^{\mathrm{II}}\right)
\end{aligned}
$$

where $[N]$ denotes the set $\{1,2, \ldots, N\}$. The Bayes risk is based on the team decision

$$
R=p_{0} c_{10} P_{E}^{\mathrm{I}}+\left(1-p_{0}\right) c_{01} P_{E}^{\mathrm{II}} .
$$

In Agent $a$ 's point of view

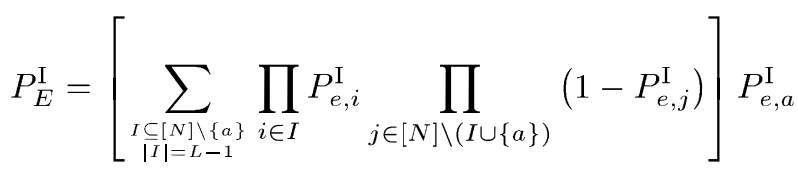

$$
\begin{aligned}
& +\sum_{n=L}^{N-1} \sum_{\substack{I \subseteq[N] \backslash\{a\} \\
|I|=n}} \prod_{i \in I} P_{e, i}^{\mathrm{I}} \prod_{j \in[N] \backslash(I \cup\{a\})}\left(1-P_{e, j}^{\mathrm{I}}\right)
\end{aligned}
$$

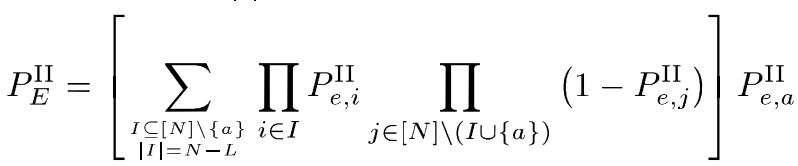

$$
\begin{aligned}
& +\sum_{n=N-L+1}^{N-1} \sum_{\substack{I \subseteq[N] \backslash\{a\} \\
|I|=n}} \prod_{i \in I} P_{e, i}^{\mathrm{II}} \prod_{j \in[N] \backslash(I \cup\{a\})}\left(1-P_{e, j}^{\mathrm{II}}\right) .
\end{aligned}
$$

The last terms in both formulas are independent of Agent $a$ 's strategy. Hence, upon observing $Y_{a}=y_{a}$, for the purpose of minimizing the Bayes risk, Agent $a$ adopts the following likelihood ratio test as its decision rule: see (4) at the bottom of the page.

Consider the special case of agents making observations through additive Gaussian noise. That is, being in state $h_{m}$ sends a signal $s_{m} \in \mathbb{R}$ to all agents, but Agent $a$ receives the corrupted signal $Y_{a}=s_{m}+W_{a}$, where the noise $W_{a}$ is assumed to be i.i.d. Gaussian with zero mean and variance $\sigma_{a}^{2}$. Then the left-hand side of (4) is given by

$$
\frac{f_{Y_{a} \mid H}\left(y_{a} \mid h_{1}\right)}{f_{Y_{a} \mid H}\left(y_{a} \mid h_{0}\right)}=\exp \left[\frac{\left(s_{1}-s_{0}\right) y_{a}}{\sigma_{a}^{2}}-\frac{s_{1}^{2}-s_{0}^{2}}{2 \sigma_{a}^{2}}\right]
$$

and the monotonicity of this expression implies that the likelihood ratio test can be simplified to a decision rule with a decision threshold $\lambda_{a}$

$$
y_{a} \sum_{\hat{H}_{a}\left(y_{a}\right)=h_{0}}^{\hat{H}_{a}\left(y_{a}\right)=h_{1}} \lambda_{a} .
$$

Hereafter we will assume that the agents have identically-distributed observations. Along with the use of the $L$-out-of- $N$ rule for fusion, this gives the agents symmetric roles. The likelihood ratio test (4) becomes identical for each agent, and in the case that the likelihood ratio is monotonic, the threshold in (5) becomes identical.

Limiting attention to identical decision rules considerably simplifies the problem. Using identical local decision rules is asymptotically optimum for the binary hypothesis testing problem [25]. Furthermore, numerical experience shows that the optimal fusion rule has the $L$-out-of- $N$ form and constraining to identical decision rules results in little or no loss of performance for finite $N$ [16]. These results have been obtained in decentralized detection models in which the fusion rule is to be optimized in addition to the local decision rules. Our model has a fixed $L$-out-of- $N$ fusion rule and only local decision rules are subject to optimization. Numerical experiments show that the restriction to identical decision rules leads to no loss of performance for $N \leq 5$ in the Gaussian likelihoods case (see Sections V-A and V-B) and the exponential likelihoods case (see Section V-C). Thus, we constrain the agents to use identical decision rules in the remainder of the paper. In particular, coordination as described in the following section enables the use of the identical decision rules in Section IV-C.

\section{Quantization of Prior Probabilities}

We generalize the classical formulation in which all agents know the true prior probability $p_{0}=\mathbb{P}\left(H=h_{0}\right)$ by assuming each Agent $i$ bases its decision rule on $q_{i}\left(p_{0}\right)$, where $q_{i}$ a scalar quantizer on $[0,1]$ (see Fig. 1). Such a setting can arise when

$$
\frac{f_{Y_{a} \mid H}\left(y_{a} \mid h_{1}\right)}{f_{Y_{a} \mid H}\left(y_{a} \mid h_{0}\right)} \hat{H}_{\hat{H}_{a}\left(y_{a}\right)=h_{0}\left(y_{a}\right)=h_{1}} \frac{p_{0} c_{10} \sum_{\substack{I \subseteq[N] \backslash\{a\} \\ \mid I I=L-1}} \prod_{i \in I} P_{e, i}^{\mathrm{I}} \prod_{j \in[N] \backslash(I \cup\{a\})}\left(1-P_{e, j}^{\mathrm{I}}\right)}{\left(1-p_{0}\right) c_{01} \sum_{\substack{I \subseteq[N] \backslash\{a\} \\|I|=N-L}} \prod_{i \in I} P_{e, i}^{\mathrm{II}} \prod_{j \in[N] \backslash(I \cup\{a\})}\left(1-P_{e, j}^{\mathrm{II}}\right)} .
$$




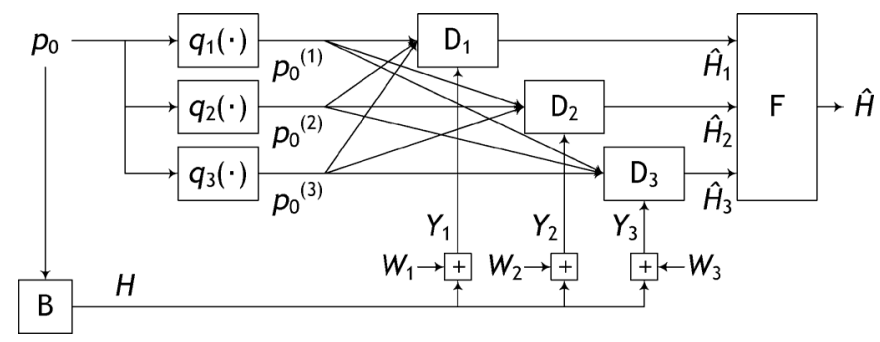

Fig. 1. A schematic diagram depicting the problem information pattern for $N=3$. The environment B generates a Bernoulli signal $H$. Its prior probability $p_{0}$ is quantized by three separate quantizers; the results are used by local agents $\mathrm{D}_{i}$. Each agent also has access to $H$ corrupted by i.i.d. noise $W_{i}$. The fusion center $\mathrm{F}$ determines $\hat{H}$ from the local decisions $\hat{H}_{i}$.

the team faces an ensemble of binary hypothesis testing problems and the agents lack the ability to have a different decision rule for each problem instance. The prior probability $p_{0}$ is thus modeled as a realization of a random variable $P_{0}$ with density function $f_{P_{0}}$. This section discusses computation of Bayes risk averaged over $P_{0}$ and the optimization of the quantizers applied to the prior probability, with $f_{P_{0}}$ given.

To optimize team performance, all agents must coordinate by sharing their quantized prior probabilities. The following steps describe the collaborative decision making process.

1) Quantizer design: For the given $f_{P_{0}}$, the $K$-level quantizers $q_{1}, \ldots, q_{N}$ are designed. These quantizers remain fixed for all time.

2) Coordination: Agents encounter an object whose prior probability is $p_{0}$. Each agent applies its quantizer to the prior probability and sends the output $q_{i}\left(p_{0}\right)$ to all other agents as a part of collaboration.

3) Decision rule design: Agents determine the best common decision rule based on $q_{1}\left(p_{0}\right), \ldots, q_{N}\left(p_{0}\right)$.

4) Signal observation and decision making: Each agent observes a noisy signal $Y_{i}$ and applies the decision rule to make a local decision $\hat{H}_{i}$.

5) Decision fusion: All local decisions $\hat{H}_{i}$ are fused to produce a global decision $\hat{H}$.

Quantizer design and decision rule design are detailed in this section. The operations of local decision making and global decision fusion have already been discussed. Coordination simply involves communication.

The Bayes risk incurred when each agent bases its local decision on its quantized prior probability is denoted $R_{M}\left(q_{1}\left(p_{0}\right), \ldots, q_{N}\left(p_{0}\right)\right) .{ }^{1}$ This is called mismatched Bayes risk to contrast to the true Bayes risk $R\left(p_{0}\right)$ if the agents know the true prior probability $p_{0}$. The Bayes risk error is defined as

$d\left(p_{0}, q_{1}\left(p_{0}\right), \ldots, q_{N}\left(p_{0}\right)\right)=R_{M}\left(q_{1}\left(p_{0}\right), \ldots, q_{N}\left(p_{0}\right)\right)-R\left(p_{0}\right)$.

We compute the mean Bayes risk error (MBRE) as the distortion of the quantizers for prior probabilities, which measures the average performance over all $p_{0}$

$$
\begin{aligned}
D & =\mathbb{E}\left[d\left(P_{0}, q_{1}\left(P_{0}\right), \ldots, q_{N}\left(P_{0}\right)\right]\right. \\
& =\int_{0}^{1} d\left(p_{0}, q_{1}\left(p_{0}\right), \ldots, q_{N}\left(p_{0}\right)\right) f_{P_{0}}\left(p_{0}\right) d p_{0} .
\end{aligned}
$$

${ }^{1}$ Note that by coordination, each agent knows other agents' quantized prior probabilities.
Minimum MBRE quantizers are to be designed within this model.

\section{A. Single Agent}

Let us first review the case of $N=1$ [7]. The optimal choice of $K$ values for quantized prior probabilities is described by the minimum MBRE quantizer

$$
q_{1}^{*}=\underset{q_{1}}{\arg \min } \mathbb{E}\left[d\left(P_{0}, q_{1}\left(P_{0}\right)\right)\right],
$$

where the distortion measure of the single quantizer $q_{1}$ is the Bayes risk error

$$
\begin{aligned}
d\left(p_{0},\right. & \left.q_{1}\left(p_{0}\right)\right)=R_{M}\left(q_{1}\left(p_{0}\right)\right)-R\left(p_{0}\right) \\
= & p_{0} c_{10} P_{E}^{\mathrm{I}}\left(q_{1}\left(p_{0}\right)\right)+\left(1-p_{0}\right) c_{01} P_{E}^{\mathrm{II}}\left(q_{1}\left(p_{0}\right)\right) \\
& -p_{0} c_{10} P_{E}^{\mathrm{I}}\left(p_{0}\right)-\left(1-p_{0}\right) c_{01} P_{E}^{\mathrm{II}}\left(p_{0}\right) .
\end{aligned}
$$

Theorem 1 ([7, Thm. 1]): The Bayes risk error $d\left(p_{0}, a\right)$ is nonnegative and only equal to zero when $p_{0}=a$. As a function of $p_{0} \in(0,1)$, it is continuous and strictly convex for all $a$.

Theorem 2 ([7, Thm. 2]): For any deterministic likelihood ratio test $\hat{H}(\cdot)$, as a function of $a \in(0,1)$ for all $p_{0}$, the Bayes risk error $d\left(p_{0}, a\right)$ has exactly one stationary point, which is a minimum.

Due to the strict convexity of $d\left(p_{0}, a\right)$ in $p_{0}$ for all $a$, quantizers that satisfy necessary conditions for MBRE optimality are regular; quantization cells are subintervals $\mathcal{R}_{1}=\left[0, b_{1}\right), \mathcal{R}_{2}=$ $\left[b_{1}, b_{2}\right), \ldots, \mathcal{R}_{K}=\left[b_{k-1}, 1\right]$, and each representation point $a_{k}$ is in $\mathcal{R}_{k}$. The necessary conditions for the optimality of a quantizer for $f_{P_{0}}\left(p_{0}\right)$ are now described.

A nearest neighbor condition describes an expression for the cell boundaries $\left\{b_{k}\right\}$ for fixed representation points $\left\{a_{k}\right\}$. Between two consecutive representation points $a_{k}$ and $a_{k+1}$, the cell boundary $b_{k}$ needs to separate $x_{1}$ such that $d\left(x_{1}, a_{k}\right)<$ $d\left(x_{1}, a_{k+1}\right)$ and $x_{2}$ such that $d\left(x_{2}, a_{k}\right)>d\left(x_{2}, a_{k+1}\right)$. Thus, $b_{k}=b^{*}$ is obtained from

$$
d\left(b^{*}, a_{k}\right)=d\left(b^{*}, a_{k+1}\right) .
$$

The point is found to be

$b^{*}$

$$
=\frac{c_{01}\left(P_{E}^{\mathrm{II}}\left(a_{k+1}\right)-P_{E}^{\mathrm{II}}\left(a_{k}\right)\right)}{c_{01}\left(P_{E}^{\mathrm{II}}\left(a_{k+1}\right)-P_{E}^{\mathrm{II}}\left(a_{k}\right)\right)-c_{10}\left(P_{E}^{\mathrm{I}}\left(a_{k+1}\right)-P_{E}^{\mathrm{I}}\left(a_{k}\right)\right)} .
$$

A centroid condition describes optimal representation points for fixed quantization cells. The MBRE is expressed as the sum of integrals over quantization regions

$$
D=\sum_{k=1}^{K} \int_{\mathcal{R}_{k}} d\left(p_{0}, a_{k}\right) f_{P_{0}}\left(p_{0}\right) d p_{0},
$$

and the minimization may be performed for each cell separately. The representation point $a_{k}=a^{*}$ of the cell $\mathcal{R}_{k}$ is chosen from the optimization problem

$$
a^{*}=\underset{a}{\arg \min }\left\{\int_{\mathcal{R}_{k}} d\left(p_{0}, a\right) f_{P_{0}}\left(p_{0}\right) d p_{0}\right\} .
$$

Since Bayes risk error is a Bregman divergence [6], the unique minimizer is the centroid of the region $\mathcal{R}_{k}$

$$
a^{*}=\frac{\int_{\mathcal{R}_{k}} p_{0} f_{P_{0}}\left(p_{0}\right) d p_{0}}{\int_{\mathcal{R}_{k}} f_{P_{0}}\left(p_{0}\right) d p_{0}} .
$$


The minimum MBRE quantizer $q_{1}^{*}$ can be found by the iterative Lloyd-Max algorithm, which alternates between the nearest neighbor and the centroid conditions.

\section{B. Identical Quantizers}

Consider, for $N>1$, that all agents use the same quantizer $q$ for prior probabilities. For an object with prior probability $p_{0}$, all agents use $p^{\prime}=q\left(p_{0}\right)$ as the object's prior. The agents will incur the mismatched Bayes risk

$$
R_{\mathrm{M}}=p_{0} c_{10} P_{E}^{\mathrm{I}}+\left(1-p_{0}\right) c_{01} P_{E}^{\mathrm{II}}
$$

due to their decisions, but what they minimize is perceived Bayes risk

$$
R_{\mathrm{P}}=p^{\prime} c_{10} P_{E}^{\mathrm{I}}+\left(1-p^{\prime}\right) c_{01} P_{E}^{\mathrm{II}}
$$

The probabilities $P_{E}^{\mathrm{I}}$ and $P_{E}^{\mathrm{II}}$ in (7) and (8) are identical. In other words, $P_{E}^{\mathrm{I}}$ and $P_{E}^{\mathrm{II}}$ are determined from the decision rules that minimize the perceived Bayes risk (8) and applied to compute the mismatched Bayes risk (7).

A general way to find local decision rules is to directly optimize (1) and (2), but these are generally complicated functions. One way to simplify the analysis for additive noise observation models is to find a decision-making model with a single agent whose performance is the same as the original team with multiple agents. By the same performance, we mean that, for the same prior-probability quantizer and decision rule, the Type I and Type II error probabilities of the new single agent are equal to the team error probabilities $P_{E}^{\mathrm{I}}$ and $P_{E}^{\mathrm{II}}$ of the original team. Thus, their optimal decision rules are also identical.

Theorem 3: Assume that the unquantized prior probability is known. Consider $N$ agents that perform group decision-making with observations corrupted by additive noises $W_{1}, \ldots, W_{N}$. For convenience, index the agents in descending order of the realizations of the noises: $W_{(1)} \geq W_{(2)} \geq \cdots \geq W_{(N)}$. When their decisions are fused by the $L$-out-of- $N$ rule, their performance is the same as that of a single agent having the same Bayes costs if its observation is corrupted by the $L$ th largest additive noise $V=W_{(L)}$.

Proof: The global decision is the same as the decision of Agent $L$ because all agents adopt the same decision threshold $\lambda$. If Agent $L$ declares $h_{0}$, then Agents $L+1, \ldots, N$, whose observations are smaller than or the same as that of Agent $L$, also declare $h_{0}$. Since at least $N-L+1$ agents send $h_{0}$, the fusion rule gives $h_{0}$ as the global decision. If Agent $L$ declares $h_{1}$, then Agents $1, \ldots, L-1$, whose observations are larger than or at least the same as that of Agent $L$, also declare $h_{1}$ and the global decision is $h_{1}$.

As such, the Bayes risk (3) can be rewritten as

$$
\begin{aligned}
R= & p_{0} c_{10} P_{E}^{\mathrm{I}}+\left(1-p_{0}\right) c_{01} P_{E}^{\mathrm{II}} \\
= & p_{0} c_{10} \mathbb{P}\left(W_{(L)}+s_{0} \geq \lambda \mid H=h_{0}\right) \\
& \quad+\left(1-p_{0}\right) c_{01} \mathbb{P}\left(W_{(L)}+s_{1}<\lambda \mid H=h_{1}\right) .
\end{aligned}
$$

If we consider a new single-agent problem with additive noise $V=W_{(L)}$, then the Bayes risk of the single agent is equal to
(9). Therefore, the optimal decision rule of the single agent is equal to that of the multiple agents, and the single agent obtains the same performance as the team of multiple agents.

When the $W_{i}$ are i.i.d. continuous random variables with pdf $f_{W}$ and cdf $F_{W}$, the random variable $V=W_{(L)}$ is well understood from the theory of order statistics [26]. The pdf of $V$ is

$f_{V}(v)=\frac{N !}{(N-L) !(L-1) !} F_{W}^{N-L}(v)\left[1-F_{W}(v)\right]^{L-1} f_{W}(v)$.

Thus, we only need to consider a single agent with a different additive noise $V$, no matter how many agents there are. The Bayesian decision rule of the single agent that observes $Y=$ $s_{m}+V$ is given by

$$
\frac{f_{Y \mid H}\left(y \mid h_{1}\right)}{f_{Y \mid H}\left(y \mid h_{0}\right)}=\frac{f_{V}\left(y-s_{1}\right)}{f_{V}\left(y-s_{0}\right)}{\stackrel{\hat{H}}{\hat{H}(y)=h_{0}}}_{\gtrless} \frac{p_{0} c_{10}}{\left(1-p_{0}\right) c_{01}}
$$

which can also be used by the multiple agents.

Hence, for the multiple agents that know quantized prior probability $q\left(p_{0}\right)$, their decision rule is given by

$$
\frac{f_{V}\left(y_{i}-s_{1}\right)}{f_{V}\left(y_{i}-s_{0}\right)} \hat{H}_{\hat{H}_{i}}{\left.\stackrel{\hat{H}_{i}}{(}\left(y_{i}\right)=h_{0}\right)=h_{1}}_{\gtrless}^{\gtrless} \frac{q\left(p_{0}\right) c_{10}}{\left(1-q\left(p_{0}\right)\right) c_{01}} .
$$

Theorem 4: If multiple agents use identical quantizers for prior probabilities, then their optimal quantizer is equal to the optimal quantizer in the equivalent single-agent model.

Proof: Since Theorem 3 is valid for any prior probability, Theorem 3 holds for quantized prior probabilities whenever the multiple agents and the single agent quantize the prior probability to the same value. Thus, their average performances are also the same if they use the same quantizer $q$ for prior probability. As a result, the single agent can achieve the minimum MBRE by adopting the optimal quantizer of the multiple agents and vice versa.

The analysis and design of the minimum MBRE quantizer in a single-agent model in [7] can be applied to the multiple-agent model without any change except the noise model.

\section{Diverse Quantizers}

Now consider the setting where each agent may have its own quantizer for prior probabilities. For an object with prior probability $p_{0}$, Agent $i$ believes that the probability of the object being in state $h_{0}$ is $p^{(i)}=q_{i}\left(p_{0}\right)$, which may be different from Agent $j$ 's quantization $p^{(j)}=q_{j}\left(p_{0}\right)$. This diversity may allow the agents to improve the quality of their decisions as a team.

The coordination phase of the collaborative decision making process is key in deriving maximum advantage from the diversity of the agents. When agents use identical quantizers, all agents consider the same perceived Bayes risk (8) even if they do not communicate, but here collaboration must be established.

Agents may try to minimize their own perceived Bayes risks so as to give the best decisions for all agents. When all agents quantize the prior probability to different values, however, they have different perceived Bayes risks:

$$
R_{\mathrm{P}}^{(i)}=p^{(i)} c_{10} P_{E}^{\mathrm{I}}+\left(1-p^{(i)}\right) c_{01} P_{E}^{\mathrm{II}} \neq R_{\mathrm{P}}^{(j)} .
$$


If all agents individually optimize their decision rules, the resulting performance is not as good as their best together [11].

All agents will incur the same cost as a result of their decisions: $c_{10}$ if their team misreads $h_{0}$ as $h_{1}$ and $c_{01}$ if their team misreads $h_{1}$ as $h_{0}$. This motivates them to collaborate by sharing a common goal that replaces their individual perceived Bayes risks. As the common goal, we introduce a weighted sum of the perceived Bayes risks called perceived common risk

$$
R_{\mathrm{PC}}=\sum_{i=1}^{N} u_{i} R_{\mathrm{P}}^{(i)}
$$

where $u_{i}$ are known constants that satisfy $\sum_{i=1}^{N} u_{i}=1$ and $u_{i}>0$ for all $i \in[N]$. The $u_{i}$ values may reflect hierarchy (power) or confidence of agents. The decision rule that is determined based on the perceived common risk is given by

$$
\frac{f_{V}\left(y_{i}-s_{1}\right)}{f_{V}\left(y_{i}-s_{0}\right)} \hat{H}_{\hat{H}_{i}\left(y_{i}\right)}{\left.\stackrel{(y)}{i}=h_{0}\right)=h_{1}}^{\gtreqless} \frac{\sum_{j=1}^{N} u_{j} q_{j}\left(p_{0}\right) c_{10}}{\left(1-\sum_{j=1}^{N} u_{j} q_{j}\left(p_{0}\right)\right) c_{01}} .
$$

The introduction of the perceived common risk allows us to treat identical-quantizer and diverse-quantizer settings on a common footing. The minimum perceived-common-risk decision rule can be used since, if all agents use identical quantizers like in Section IV-B, their perceived common risk is equal to their perceived Bayes risks:

$$
\begin{aligned}
R_{\mathrm{PC}} & =\sum_{i=1}^{N} u_{i}\left(p^{(i)} c_{10} P_{E}^{\mathrm{I}}+\left(1-p^{(i)}\right) c_{01} P_{E}^{\mathrm{II}}\right) \\
& =\sum_{i=1}^{N} u_{i}\left(p^{\prime} c_{10} P_{E}^{\mathrm{I}}+\left(1-p^{\prime}\right) c_{01} P_{E}^{\mathrm{II}}\right) \\
& =p^{\prime} c_{10} P_{E}^{\mathrm{I}}+\left(1-p^{\prime}\right) c_{01} P_{E}^{\mathrm{II}}=R_{\mathrm{P}} .
\end{aligned}
$$

The decision rules that are determined based on the perceived common risk are generally not optimal for a given $p_{0}$. Specifically, the decision rules are optimal if and only if $p_{0}=\sum_{i=1}^{n} u_{i} p^{(i)}$. However, this suboptimality is due to the quantization of the prior probabilities, not because the perceived common risk is a bad criterion. The performance of the decision-making team is measured by the Bayes risk averaged over $P_{0}$, not by the Bayes risk for specific $p_{0}$. Thus, a set of quantizers that leads to good decisions on average is essential for the agents to use the perceived common risk as a common goal of minimization.

Design of such quantizers has high computational complexity. In Section IV-B, a single quantizer is easily designed by the iterative Lloyd-Max algorithm, which utilizes independence among endpoints of quantization cells in the nearest neighbor step (optimizing cell boundaries) and independence among representation points in the centroid step (optimizing representation points). On the other hand, multiple quantizers that define different quantization cells do not have such independencies.

For the simple example of Fig. 2, the cell $\mathcal{R}_{21}$ of the quantizer $q_{2}$ affects the decisions for objects whose prior probabilities are within the interval $\mathcal{C}_{1}$ or $\mathcal{C}_{2}$. The team performance in

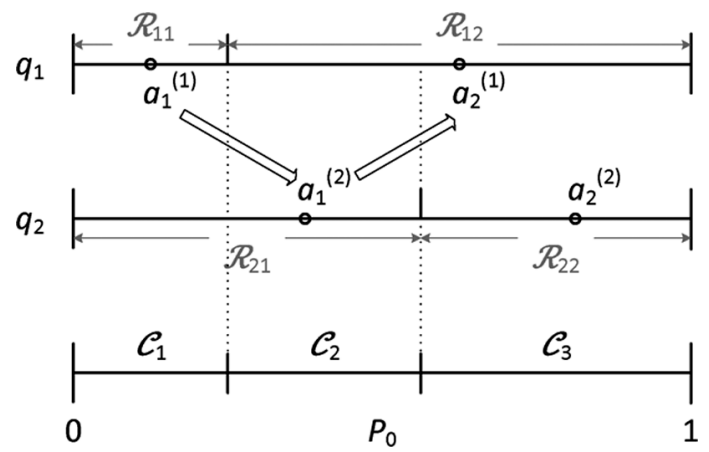

Fig. 2. An example that shows how the dependency among representation points propagates to break the independence between representation points of two different cells $\mathcal{R}_{11}$ and $\mathcal{R}_{12}$.

the interval $\mathcal{C}_{1}$ is affected by the representation points $a_{1}^{(1)}$ and $a_{1}^{(2)}$ and by $a_{2}^{(1)}$ and $a_{1}^{(2)}$ in the interval $\mathcal{C}_{2}$. Hence, we can observe that dependency is propagated through the representation points: a choice of $a_{2}^{(1)}$ depends on a choice of $a_{1}^{(2)}$, which depends on a choice of $a_{1}^{(1)}$. In order to avoid such complexity, we introduce an indirect method to optimize diverse quantizers.

Consider a team of $N$ agents that respectively use different $K$-level quantizers $q_{1}, \ldots, q_{N}$ and another team of $N$ agents that all use an identical $K_{S}$-level quantizer $q_{S}$. Please note that each team uses identical decision rules that are determined to minimize the perceived common risks.

Theorem 5: A set of $N$ different $K$-level quantizers $\left\{q_{1}, \ldots, q_{N}\right\}$ and a $K_{S}$-level quantizer $q_{S}$ result in the same perceived common risk if, for all $p_{0} \in[0,1]$

$$
\sum_{i=1}^{N} u_{i} q_{i}\left(p_{0}\right)=q_{S}\left(p_{0}\right) .
$$

Proof: For an object with prior probability $p_{0}$, agents respectively using $\left\{q_{1}, \ldots, q_{N}\right\}$ make decisions based on individually quantized prior probabilities $p^{(i)}=q_{i}\left(p_{0}\right)$. Their perceived common risk is

$$
\begin{aligned}
R_{\mathrm{PC}, 1} & =\sum_{i=1}^{N} u_{i}\left(p^{(i)} c_{10} P_{E}^{\mathrm{I}}+\left(1-p^{(i)}\right) c_{01} P_{E}^{\mathrm{II}}\right) \\
& =\left(\sum_{i=1}^{N} u_{i} p^{(i)}\right) c_{10} P_{E}^{\mathrm{I}}+\left(\sum_{i=1}^{N} u_{i}\left(1-p^{(i)}\right)\right) c_{01} P_{E}^{\mathrm{II}}
\end{aligned}
$$

For agents using the common quantizer $q_{S}$, with quantized prior probability $p^{\prime}=q_{S}\left(p_{0}\right)$, their perceived common risk is

$$
R_{\mathrm{PC}, 2}=p^{\prime} c_{10} P_{E}^{\mathrm{I}}+\left(1-p^{\prime}\right) c_{01} P_{E}^{\mathrm{II}} .
$$

The perceived common risks (13) and (14) only depend on the decision rules used by $N$ agents. If there exists a positive constant $t$ that satisfies

$$
R_{\mathrm{PC}, 1}=t \cdot R_{\mathrm{PC}, 2}
$$

for any $N$-tuple of decision rules, then the $N$-tuple that minimizes $R_{\mathrm{PC}, 1}$ also minimizes $R_{\mathrm{PC}, 2}$. If the proportionality 
property holds for all $p_{0} \in[0,1]$, then the agents in both cases always use the same decision rules. Therefore, the set of different quantizers $\left\{q_{1}, \ldots, q_{N}\right\}$ and the set of identical quantizers $\left\{q_{S}, \ldots, q_{S}\right\}$ will cause the same Bayes risk on any problem in the ensemble.

For any $N$-tuple and any $p_{0}, R_{\mathrm{PC}, 1}=t \cdot R_{\mathrm{PC}, 2}$ if and only if $\sum_{i=1}^{N} u_{i} p^{(i)}=t p^{\prime}$ and $\sum_{i=1}^{N} u_{i}\left(1-p^{(i)}\right)=t\left(1-p^{\prime}\right)$. From the fact that $\sum_{i=1}^{N} u_{i}=1$, the constant $t$ can only be 1 , and the condition is simplified to

$$
\sum_{i=1}^{N} u_{i} q_{i}\left(p_{0}\right)=q_{S}\left(p_{0}\right), \quad \text { for all } p_{0} \in[0,1]
$$

This simple condition comes from the fact that the perceived common risk is a weighted sum of the perceived Bayes risks.

Theorem 6: A team of $N$ agents individually using diverse $K$-level regular quantizers can achieve the minimum mean Bayes risk error that they can achieve when they use the same $(N(K-1)+1)$-level quantizer.

Proof: When the agents use an identical $K_{S}$-level quantizer, the optimal quantizer $q_{S}$ is always a regular quantizer [7]; each quantization cell $\mathcal{C}_{k}$ is an interval and its representation point $x_{k}$ is within the interval. Equation (12) is given by

$$
q_{S}\left(p_{0}\right)=\sum_{i=1}^{N} u_{i} q_{i}\left(p_{0}\right)
$$

In a cell $\mathcal{C}_{k}, q_{S}\left(p_{0}\right)=x_{k}$ and $q_{i}\left(p_{0}\right)=a^{(i)}$ are constants, where $a^{(i)}$ is one of the representation points of $K$-level quantizer $q_{i}$, $i=1, \ldots, N$. Thus, the condition (12) is the same as

$$
x_{k}=\sum_{i=1}^{N} a^{(i)} u_{i}
$$

Overall, we have a total of $K_{S}$ equations, which are described in the following matrix form:

$$
\left[\begin{array}{c}
x_{1} \\
\vdots \\
x_{K_{S}}
\end{array}\right]=\mathbf{A}\left[\begin{array}{c}
u_{1} \\
\vdots \\
u_{N}
\end{array}\right]
$$

where $\mathbf{A}$ is a $K_{S} \times N$ matrix that has $\left[\begin{array}{llll}a_{k_{j 1}}^{(1)} & a_{k_{j 2}}^{(2)} & \cdots & a_{k_{j N}}^{(N)}\end{array}\right]$ as its $j$ th row and $a_{k_{j i}}^{(i)}$ is the representation point of the $k_{j i}$ th cell of $q_{i}$ for any $1 \leq k_{j i} \leq K$. The $k_{j i}$ th cell indicates the quantization cell of $q_{i}$ that includes the subcell $\mathcal{C}_{j}$.

There exists an $\mathbf{A}$ that satisfies (15) only if there exists a set of $N$ different $K$-level quantizers $q_{1}, \ldots, q_{N}$ that are equivalent to $q_{S}$. In (15), the vector $\left[\begin{array}{llll}u_{1} & u_{2} & \cdots & u_{N}\end{array}\right]^{T}$ is a given parameter and the vector $\left[\begin{array}{llll}x_{1} & x_{2} & \cdots & x_{K_{S}}\end{array}\right]^{T}$ is uniquely determined by the optimization of $q_{S}$; the matrix $\mathbf{A}$ consists of unknown parameters (i.e., representation points of $q_{i}$ ) to be determined. However, if $\mathbf{A}$ has any linearly dependent row, the representation points may not exist. On the other hand, if $\mathbf{A}$ has only linearly independent rows and $K_{S} \leq N K$, then we can solve (15) to find all the representation points of $q_{i}$.
The maximum number of linearly independent rows of $\mathbf{A}$ is $N(K-1)+1$

$$
\mathbf{A}=\left[\begin{array}{cccc}
a_{1}^{(1)} & a_{1}^{(2)} & \cdots & a_{1}^{(N)} \\
\hline a_{2}^{(1)} & a_{1}^{(2)} & \cdots & a_{1}^{(N)} \\
a_{1}^{(1)} & a_{2}^{(2)} & \cdots & a_{1}^{(N)} \\
\vdots & \vdots & \ddots & \vdots \\
a_{1}^{(1)} & a_{1}^{(2)} & \cdots & a_{2}^{(N)} \\
\hline a_{3}^{(1)} & a_{1}^{(2)} & \cdots & a_{1}^{(N)} \\
a_{1}^{(1)} & a_{3}^{(2)} & \cdots & a_{1}^{(N)} \\
\vdots & \vdots & \ddots & \vdots \\
a_{1}^{(1)} & a_{1}^{(2)} & \cdots & a_{3}^{(N)} \\
\vdots \vdots & \vdots & \ddots & \vdots \\
\hline a_{K}^{(1)} & a_{1}^{(2)} & \cdots & a_{1}^{(N)} \\
a_{1}^{(1)} & a_{K}^{(2)} & \cdots & a_{1}^{(N)} \\
\vdots & \vdots & \ddots & \vdots \\
a_{1}^{(1)} & a_{1}^{(2)} & \cdots & a_{K}^{(N)}
\end{array}\right] .
$$

Any other row $\left[\begin{array}{llll}a_{k_{1}}^{(1)} & a_{k_{2}}^{(2)} & \ldots & a_{k_{N}}^{(N)}\end{array}\right]$ is a linear combination of the $N(K-1)+1$ rows. ${ }^{2}$ Thus, the existence of $\left\{q_{1}, \ldots, q_{N}\right\}$ that is equivalent to $q_{S}$ is only guaranteed when $K \leq K_{S} \leq$ $N(K-1)+1$.

A quantizer with greater $K_{S}$ does not increase mean Bayes risk error [7]. Therefore, the minimum mean Bayes risk error of agents using diverse $K$-level quantizers is upper bounded by that of agents using the identical $(N(K-1)+1)$-level quantizer.

The significance of Theorem 5 is that it enables a simple design algorithm for diverse quantizers $q_{1}, \ldots, q_{N}$. Without loss of performance with respect to the mean Bayes risk, instead of solving the more difficult minimization problem

$$
\left(q_{1}^{*}, \ldots, q_{N}^{*}\right)=\underset{q_{1}, \ldots, q_{N}}{\arg \min } \mathbb{E}\left[d\left(P_{0}, q_{1}\left(P_{0}\right), \ldots, q_{N}\left(P_{0}\right)\right)\right]
$$

we can solve the simpler minimization problem

$$
q_{S}^{*}=\underset{q_{S}}{\arg \min } \mathbb{E}\left[d\left(P_{0}, q_{S}\left(P_{0}\right), \ldots, q_{S}\left(P_{0}\right)\right)\right]
$$

and find $q_{1}^{*}, \ldots, q_{N}^{*}$ that, for all $p_{0}$, satisfy (12)

$$
\sum_{i=1}^{N} u_{i} q_{i}^{*}\left(p_{0}\right)=q_{S}^{*}\left(p_{0}\right) .
$$

Furthermore, even the design of $q_{S}$ in (17) is simplified by Theorem 4 to a single-agent, single-quantizer problem, where the quantizer has $N(K-1)+1$ cells according to Theorem 6 .

The optimal set of $K$-level regular quantizers for $N$ agents is always a set of diverse quantizers if $K>1$. The optimal set of quantizers can be designed by a two-step algorithm. The first step is to design the optimal $(N(K-1)+1)$-level quantizer $q_{S}$ to be commonly used by $N$ agents, e.g., with the Lloyd-Max algorithm [7].

The second step is to disassemble the quantizer $q_{S}$ into $N$ different $K$-level regular quantizers $q_{1}, \ldots, q_{N}$ that lead to the

${ }^{2}$ For example, $\left[\begin{array}{lllllll}a_{1}^{(1)} & a_{2}^{(2)} & a_{2}^{(3)} & a_{1}^{(4)} & a_{1}^{(5)} & \ldots & a_{1}^{(N)}\end{array}\right]$ is the second row of $\mathbf{A}$ plus the third row minus the first row. 
same mean Bayes risk. As mentioned after Theorem 2, $q_{S}$ is a regular quantizer. We are considering only the construction of regular quantizers $q_{1}, \ldots, q_{N}$; nonregular quantizers are not plausible as a model for human behavior. Since the $K$-level quantizer $q_{i}$ is regular, its quantization cells are intervals, i.e., the cell $\mathcal{R}_{i k}$ is defined to be $\left[b_{k-1}^{(i)}, b_{k}^{(i)}\right)$ for any Agent $i$ and cell index $1 \leq k \leq K$.

In order to satisfy (12), each cell boundary of $q_{S}$ needs to be a cell boundary of at least one of $q_{1}, \ldots, q_{N}$ because, for any cell boundary $y$ of $q_{S}$ and for any $x_{1}<y$ and $x_{2}>y$, $\sum_{i=1}^{N} u_{i} q_{i}\left(x_{1}\right)=q_{S}\left(x_{1}\right) \neq q_{S}\left(x_{2}\right)=\sum_{i=1}^{N} u_{i} q_{i}\left(x_{2}\right)$. Also, any two or more quantizers out of $q_{1}, \ldots, q_{N}$ cannot have a common cell boundary since $q_{S}$ has $N(K-1)+1$ cells. As a result, each cell boundary of $q_{S}$, except 0 and 1 , needs to be a cell boundary of exactly one of $q_{1}, \ldots, q_{N}$.

Therefore, we determine cell boundaries of $q_{1}, \ldots, q_{N}$ by splitting the cell boundaries of $q_{S}$ into $N$ sets by the following conditions:

$$
\begin{aligned}
\bigcup_{i=1}^{N} B_{i} & =B_{S}, \\
B_{i} \cap B_{j} & =\{0,1\}, \quad \text { for all } i \text { and all } j \neq i
\end{aligned}
$$

where $B_{S}$ is the set of cell boundaries of $q_{S}$ and $B_{i}$ are the sets of cell boundaries of $q_{i} ;\left|B_{S}\right|=N(K-1)+2$ and $\left|B_{i}\right|=K+1$. The sets $B_{1}, \ldots, B_{N}$ that satisfy (19) are not unique.

Representation points of the cells of $q_{1}, \ldots, q_{N}$ are determined after cell boundaries of the quantizers are fixed. We have $K_{S}=N(K-1)+1$ equations of representation points of $q_{1}, \ldots, q_{N}$ that describe the condition (12). Once we find the representation points that satisfy all the equations, then the quantizers are minimum MBRE diverse quantizers for the $N$ agents. The optimal identical quantizer $q_{S}$ is always regular because the optimal quantizer for single agent is regular, but the optimal diverse quantizers $q_{1}, \ldots, q_{N}$ need not be regular.

The total number of the representation points is $N K$, but we have $N-1$ less equations than what we need to uniquely determine the representation points. Furthermore, a different arrangement of cell boundaries changes the equations and, consequently, proper representation points. Therefore, optimal diverse $K$-level quantizers are not unique; any choice leads to the same result because Theorem 5 shows that any set of the optimal quantizers causes the same mean Bayes risk error as $q_{S}$ does.

In an example of 3 agents and 2-level quantizers presented in [9], the optimization of $q_{S}$ returns a 4-level quantizer with boundary points $B_{S}=\left\{0, y_{1}, y_{2}, y_{3}, 1\right\}$ and representation points $\left\{x_{1}, x_{2}, x_{3}, x_{4}\right\}$. Let us determine the cell boundaries of $q_{1}, q_{2}$, and $q_{3}$ to be $b_{1}^{(1)}=y_{1}, b_{1}^{(2)}=y_{2}$, and $b_{1}^{(3)}=y_{3}$, that is to set $B_{1}=\left\{0, y_{1}, 1\right\}, B_{2}=\left\{0, y_{2}, 1\right\}$, and $B_{3}=\left\{0, y_{3}, 1\right\}$. Then, (9) is translated to

$$
\begin{array}{ll}
u_{1} a_{1}^{(1)}+u_{2} a_{1}^{(2)}+u_{3} a_{1}^{(3)}=x_{1}, & p_{0} \in \mathcal{C}_{1}=\left[0, y_{1}\right) \\
u_{1} a_{2}^{(1)}+u_{2} a_{1}^{(2)}+u_{3} a_{1}^{(3)}=x_{2}, & p_{0} \in \mathcal{C}_{2}=\left[y_{1}, y_{2}\right) \\
u_{1} a_{2}^{(1)}+u_{2} a_{2}^{(2)}+u_{3} a_{1}^{(3)}=x_{3}, & p_{0} \in \mathcal{C}_{3}=\left[y_{2}, y_{3}\right) \\
u_{1} a_{2}^{(1)}+u_{2} a_{2}^{(2)}+u_{3} a_{2}^{(3)}=x_{4}, & p_{0} \in \mathcal{C}_{4}=\left[y_{3}, 1\right] .
\end{array}
$$

We need to solve (20) with respect to six variables $a_{1}^{(1)}, a_{2}^{(1)}, a_{1}^{(2)}, a_{2}^{(2)}, a_{1}^{(3)}$, and $a_{2}^{(3)}$, which are the represen- tation points of $q_{1}, q_{2}$, and $q_{3}$. Since we have only four equations, we can find infinitely many solutions that satisfy (20). Once we pick one, it determines $q_{1}, q_{2}$, and $q_{3}$.

\section{Comparison to Team-Oblivious Agents}

We have discussed teams of agents that are aware of the existence of the other $N-1$ agents and the $L$-out-of- $N$ fusion rule. Let us now consider the case when the agents do not know $N$ and $L$; individual agents maximize their own probability of being correct. Agent $i$ considers the Bayes risk

$$
R=p_{0} c_{10} P_{e, i}^{\mathrm{I}}+\left(1-p_{0}\right) c_{01} P_{e, i}^{\mathrm{II}} \text {. }
$$

All agents choose their quantizers as if they are single agents with additive noise $W$ drawn from $f_{W}$. Consequently, they have identical quantizers $q$ because they have the same Bayes costs $c_{10}$ and $c_{01}$.

Their quantizers are obviously not optimal. In Section IV-B, it has been shown that their optimal identical quantizers need to be designed based on the additive noise $V=W_{(L)}$, whose density function is different from $f_{W}$, in a single-agent model. Furthermore, any identical quantizers can be improved by transformation of diverse quantizers, as in Section IV-C. Therefore, for each agent, minimization of the MBRE of its own decision is not the best strategy as an optimal team member and its optimal behavior depends on $N$ and $L$. The effect of $N$ and $L$ on the design of optimal quantizers is reflected by the equivalent single-agent model in Theorem 3.

\section{E. Comparison to Uncooperative Agents}

The communication of quantized probabilities is critical in our main results; it brings in the perceived common risk and consequently enables agents to optimize their quantizers efficiently and to adopt the best decision rule. Let us briefly consider the case when the agents do not share quantized prior probabilities. Agent $a$ will use the following likelihood ratio test because $q_{a}\left(p_{0}\right)$ is its only information about prior probability:

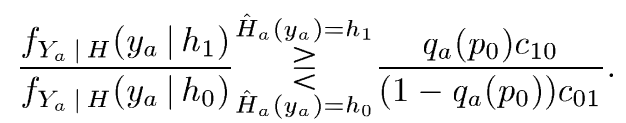

In addition, the agents cannot use perceived common risk as their decision criterion. Thus, Theorems 5 and 6 are not applicable in this case and the design of diverse quantizers becomes more complex. The resulting mean Bayes risk is also higher than that under collaboration. This case has been discussed in [27]-[29] with more details.

\section{EXAMPLES}

Throughout this section, assume that the prior probability $p_{0}$ of an object being in state $h_{0}$ is drawn from the uniform distribution $f_{P_{0}}\left(p_{0}\right)=1$ for all $p_{0} \in[0,1]$.

\section{A. Gaussian Likelihoods, MAJORITY Rule}

Suppose the object sends the signal $s_{0}=0$ in state $h_{0}$ and $s_{1}=1$ in state $h_{1}$, and each agent receives the signal corrupted by additive i.i.d. noise $W_{i}$ with $\operatorname{cdf} F_{W}$ and pdf $f_{W}$ where

$$
f_{W}(w)=\frac{1}{\sqrt{2 \pi \sigma^{2}}} e^{-w^{2} / 2 \sigma^{2}} .
$$



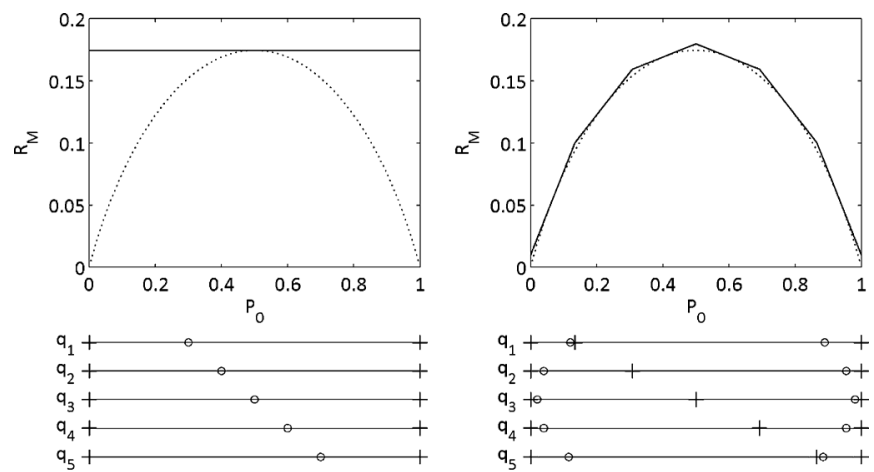

(a)
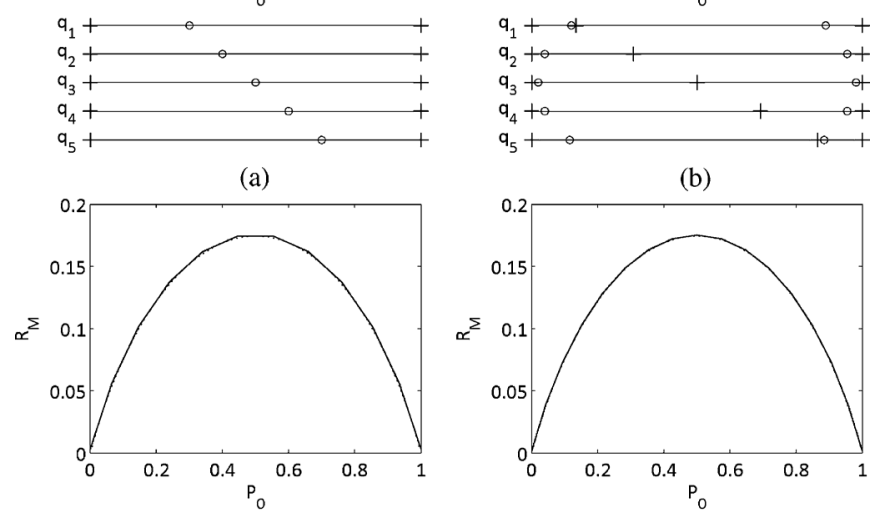

(b)
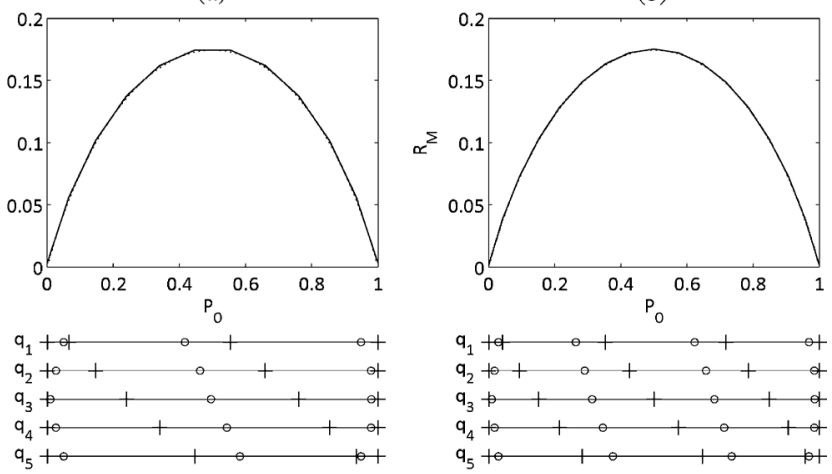

(c)

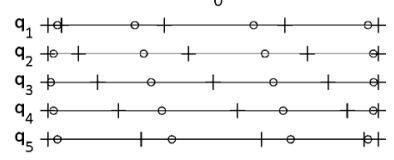

(d)

Fig. 3. Optimal diverse $K$-level quantizers (cell boundaries as + 's and representation points as o's) for uniformly distributed $P_{0}$ and the resulting mismatched Bayes risk when $N=5$ agents perform distributed hypothesis testing fused by the MAJORITY rule. The parameters are defined as $c_{10}=c_{01}=1$, $\sigma=1$, and $u_{i}=1 / 5$, for $i=1, \ldots, 5$. For comparison, the unquantized Bayes risk is depicted by dotted curves. (a) $K=1$. (b) $K=2$. (c) $K=3$. (d) $K=4$.

Each likelihood $f_{Y_{i} \mid H}$ is thus Gaussian with mean $H$. The MAJORITY rule is used to fuse local decisions made by $N$ agents. We assume that $N$ is an odd number so that voting never results in a tie.

From Theorem 3, the equivalent single-agent model has an additive noise $V_{N}^{\mathrm{MAJ}}$ with pdf given by

$f_{V_{N}^{\mathrm{MAJ}}}(v)=\frac{N !}{\left(\frac{N-1}{2} !\right)^{2}} F_{W}^{(N-1) / 2}(v)\left[1-F_{W}(v)\right]^{(N-1) / 2} f_{W}(v)$.

The mean of $V_{N}^{\mathrm{MAJ}}$ is zero and the variance of $V_{N}^{\mathrm{MAJ}}$ is proportional to that of $W_{i}$

$$
\begin{aligned}
\operatorname{var} & \left(V_{N}^{\mathrm{MAJ}}\right) \\
& =\int_{-\infty}^{\infty} v^{2} \frac{N !}{\left(\frac{N-1}{2} !\right)^{2}} F_{W}^{\frac{N-1}{2}}(v)\left[1-F_{W}(v)\right]^{\frac{N-1}{2}} f_{W}(v) d v \\
& =\int_{-\infty}^{\infty} v^{2} \frac{N !}{\left(\frac{N-1}{2} !\right)^{2}} F_{\mathcal{N}}^{\frac{N-1}{2}}\left(\frac{v}{\sigma}\right)\left[1-F_{\mathcal{N}}\left(\frac{v}{\sigma}\right)\right]^{\frac{N-1}{2}} f_{W}(v) d v \\
& =\sigma^{2} \int_{-\infty}^{\infty} z^{2} \frac{N !}{\left(\frac{N-1}{2} !\right)^{2}} F_{\mathcal{N}^{2}}^{\frac{N-1}{2}}(z)\left[1-F_{\mathcal{N}}(z)\right]^{\frac{N-1}{2}} f_{\mathcal{N}}(z) d z \\
& \triangleq \sigma^{2} \zeta_{N}^{\mathrm{MAJ}}
\end{aligned}
$$

where $f_{\mathcal{N}}$ and $F_{\mathcal{N}}$ denote the pdf and cdf of a standard normal random variable, and $z=v / \sigma$. The factor $\zeta_{N}^{\mathrm{MAJ}}$ is the variance of the median of $N$ i.i.d. standard normal random variables.

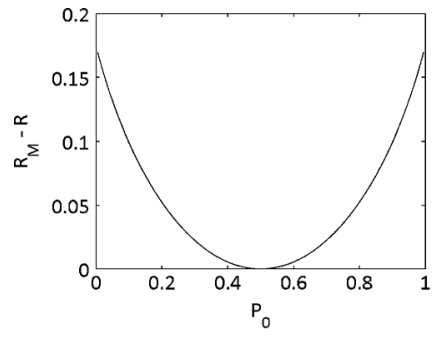

(a)

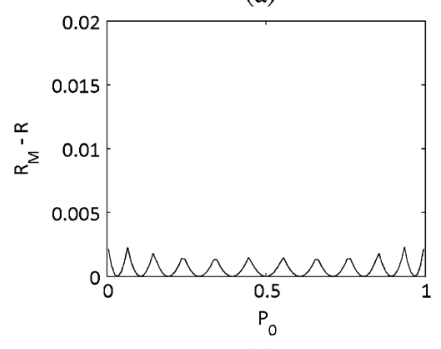

(c)

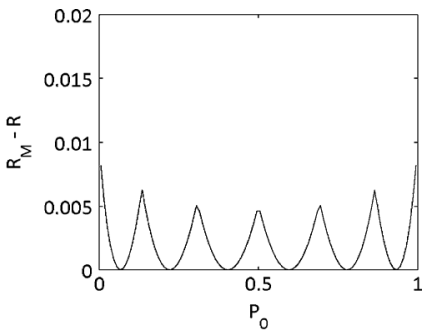

(b)

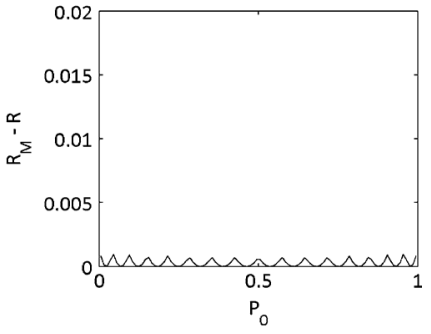

(d)
Fig. 4. Bayes risk error of the quantizers in Fig. 3. (a) $K=1$. (b) $K=2$. (c) $K=3$. (d) $K=4$.

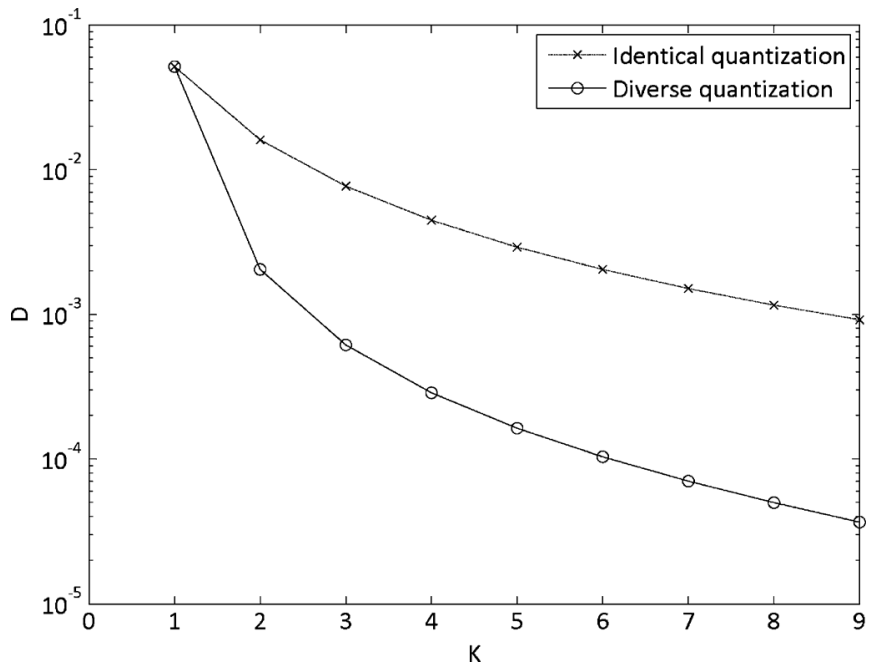

Fig. 5. Mean Bayes risk error for uniformly distributed $P_{0}$ in the example of Fig. 3.

The means and variances of Gaussian order statistics have been studied extensively [30]-[32], and the value of $\zeta_{N}^{\mathrm{MAJ}}$ for any $1 \leq N \leq 20$ can be found in [31]. For example, $\zeta_{3}^{\mathrm{MAJ}}=0.4487$ and $\zeta_{5}^{\mathrm{MAJ}}=0.2863$.

This equivalent single-agent model is considered for optimization of identical $(N(K-1)+1)$-level quantizers. An optimal set of diverse $K$-level regular quantizers for the agents can be designed from the optimal $(N(K-1)+1)$-level quantizer.

Fig. 3 shows an example of a team of five agents; 3 it depicts an optimal set of diverse $K$-level quantizers and the resulting Bayes risk for $K=1, \ldots, 4$. The Bayes risk error due to quantization of prior probabilities is depicted in Fig. 4, which shows improvement of decision making as $K$ increases. Also, the mean Bayes risk error is given in Fig. 5; it shows the advantage of using optimal diverse quantizers against using optimal identical quantizers.

\footnotetext{
${ }^{3} \mathrm{An}$ example of a team of three agents is given in [9].
} 
Asymptotically, $\zeta_{N}^{\mathrm{MAJ}}$ decays inversely with $N$; more precisely [33]

$$
\lim _{N \rightarrow \infty} N \cdot \zeta_{N}^{\mathrm{MAJ}}=\frac{\pi}{2} .
$$

If the agents could share their observations rather than their local hard decisions, the team would base its decision on the sample mean of the observations. The resulting performance is governed by the variance of the sample mean of the noise variables $W_{1}, \ldots, W_{N}$. For $\sigma=1$,

$$
\lim _{N \rightarrow \infty} N \cdot \operatorname{var}\left(N^{-1} \sum_{i=1}^{N} W_{i}\right)=1 .
$$

The ratio of $\pi / 2$ between the two asymptotic variances quantifies the loss in using majority vote rather than fusing full measurements.

\section{B. Gaussian Likelihoods, OR Rule}

Maintaining the Gaussian observation model from Section V-A, now consider fusion using the OR rule: the global decision is $h_{0}$ only when all agents declare $h_{0}$. The equivalent single-agent model has an additive noise $V_{N}^{\mathrm{OR}}$, which is the maximum order statistic of noises $W_{1}, \ldots, W_{N}$. The pdf of $V_{N}^{\mathrm{OR}}$ is given by

$$
f_{V_{N}^{\mathrm{OR}}}(v)=N F_{W}^{N-1}(v) f_{W}(v) .
$$

The mean of $V_{N}^{\mathrm{OR}}$ is proportional to $\sigma$

$$
\mathbb{E}\left[V_{N}^{\mathrm{OR}}\right]=\mu_{N}^{\mathrm{OR}} \cdot \sigma
$$

and the variance of $V_{N}^{\mathrm{OR}}$ is proportional to $\sigma^{2}$

$$
\operatorname{var}\left(V_{N}^{\mathrm{OR}}\right)=\zeta_{N}^{\mathrm{OR}} \cdot \sigma^{2}
$$

these can be proven analogously to (21). The factor $\mu_{N}^{\mathrm{OR}}$ is increasing in $N$ but $\zeta_{N}^{\mathrm{OR}}$ is decreasing in $N$. For example, $\mu_{3}^{\mathrm{OR}}=$ 0.8463 and $\mu_{5}^{\mathrm{OR}}=1.1630 ; \zeta_{3}^{\mathrm{OR}}=0.5595$ and $\zeta_{5}^{\mathrm{OR}}=0.4475$. For other $N$ between 1 and 20 , the values of $\mu_{N}^{\mathrm{OR}}$ and $\zeta_{N}^{\mathrm{OR}}$ are listed in [31].

An optimal set of diverse $K$-level regular quantizers for a team of five agents is given in Fig. 6 for $K=1, \ldots, 4$. Figs. 7 and 8 show the Bayes risk error and the mean Bayes risk error due to the quantization. The trends are similar to those in Section V-A, which considers the same model except for the fusion rule. The difference in the fusion rule changes the equivalent single-agent model. However, the same optimization algorithm of quantizers for prior probabilities can be applied in any case: designing the optimal identical $(N(K-1)+1)$-level quantizer of the equivalent single agent and disassembling the quantizer into $N$ diverse $K$-level regular quantizers. The algorithm comes from the relationship between the perceived Bayes risk and the common risk, which is defined as the weighted sum of the perceived Bayes risk. Thus, the algorithm does not depend on how the team of agents make decisions and the error probabilities $P_{E}^{\mathrm{I}}$ and $P_{E}^{\mathrm{II}}$ are computed.

Average performance with the OR rule (Fig. 8) is slightly worse than with the MAJORITY rule (Fig. 5). A qualitative understanding can be obtained through study of $V_{N}^{\mathrm{OR}}$ and $V_{N}^{\mathrm{MAJ}}$,
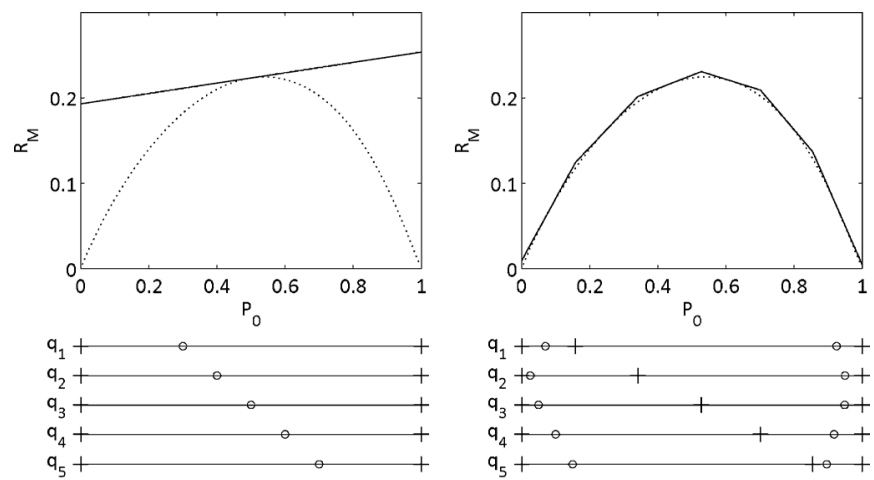

(a)

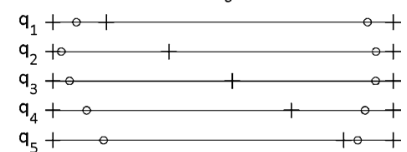

(b)
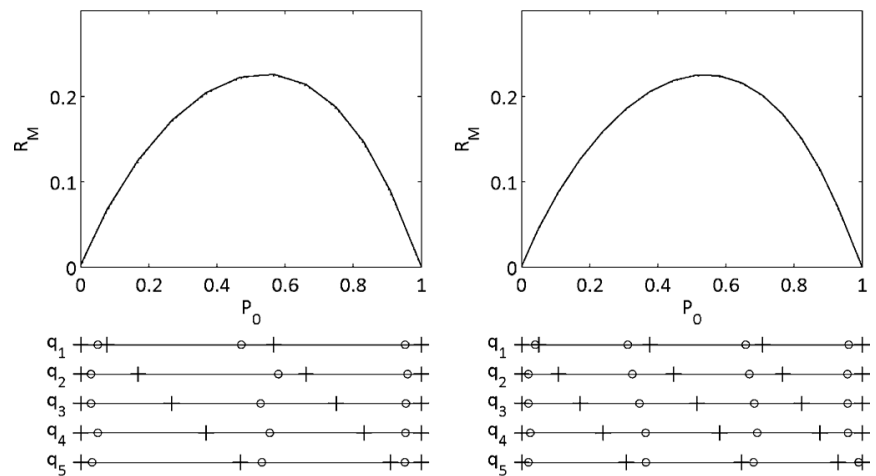

(c)

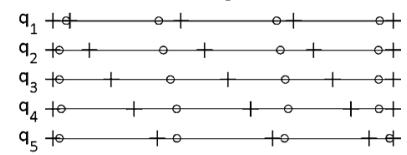

(d)

Fig. 6. Optimal diverse $K$-level quantizers (cell boundaries as + 's and representation points as $\circ$ 's) for uniformly distributed $P_{0}$ and the resulting mismatched Bayes risk when $N=5$ agents perform distributed hypothesis testing fused by the OR rule. The parameters are defined as $c_{10}=c_{01}=1, \sigma=1$, and $u_{i}=1 / 5$, for $i=1, \ldots, 5$. For comparison, the unquantized Bayes risk is depicted by dotted curves. (a) $K=1$. (b) $K=2$. (c) $K=3$. (d) $K=4$.

and asymptotic behavior of the variances of these random variables suggests that the performance gap increases with $N$.

While the mean of $V_{N}^{\mathrm{MAJ}}$ is zero for all $N, \mu_{N}^{\mathrm{OR}}$ (the mean of $V_{N}^{\mathrm{OR}}$ ) is positive for $N \geq 2$ and increasing with $N$. In the equivalent single-agent model under the OR rule, the single agent tends to observe something larger than a true signal by about $\mu_{N}^{\mathrm{OR}}$ because of the noise $V_{N}^{\mathrm{OR}}$. Hence, the optimal decision threshold of the single agent is larger than that in the model of Section V-A. This can be interpreted as any individual agent optimally requiring "stronger evidence" to declare $h_{1}$ as the number of agents increases. The optimal decision thresholds for $N=5$ are shown in Fig. 9. The asymptotic growth of $\mu_{N}^{\mathrm{OR}}$ is given by (see [26, Ex. 10.5.3])

$$
\lim _{N \rightarrow \infty} \mu_{N}^{\mathrm{OR}} /(2 \log N)^{1 / 2}=1 .
$$

If all the equivalent noise variables had the same standardized distribution, then MAJORITY and OR fusion rules for fixed $N$ could be compared through the variances $\zeta_{N}^{\mathrm{MAJ}}$ and $\zeta_{N}^{\mathrm{OR}}$. We saw in (22) that $\zeta_{N}^{\mathrm{MAJ}}=\Theta(1 / N)$. The decay of $\zeta_{N}^{\mathrm{OR}}$ is much slower; specifically (see [26, Ex. 10.5.3])

$$
\lim _{N \rightarrow \infty}\left(\frac{12}{\pi^{2}} \log N\right) \cdot \zeta_{N}^{\mathrm{OR}}=1 .
$$

This suggests that for large $N$ (and the Gaussian likelihood case), the MAJORITY rule is more effective than the OR rule. 


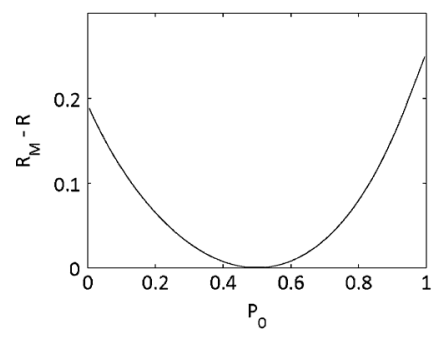

(a)

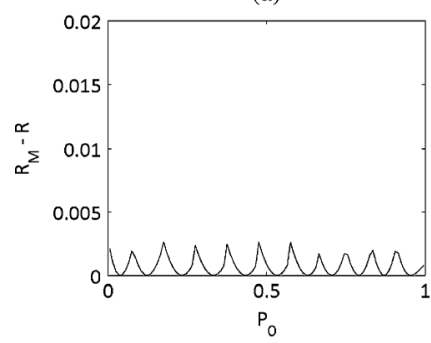

(c)

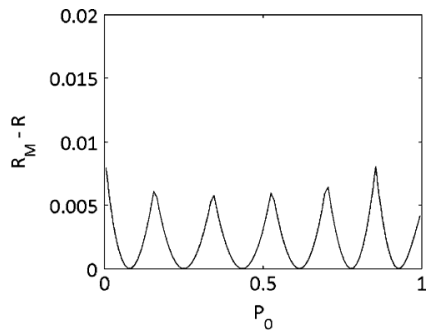

(b)

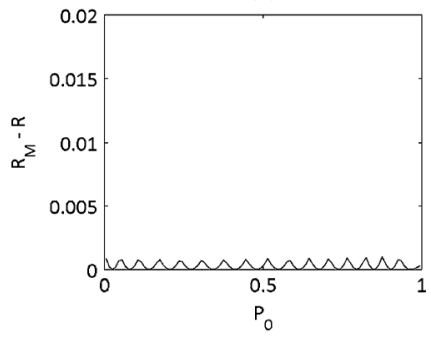

(d)
Fig. 7. Bayes risk error of the quantizers in Fig. 6. (a) $K=1$. (b) $K=2$. (c) $K=3$. (d) $K=4$.

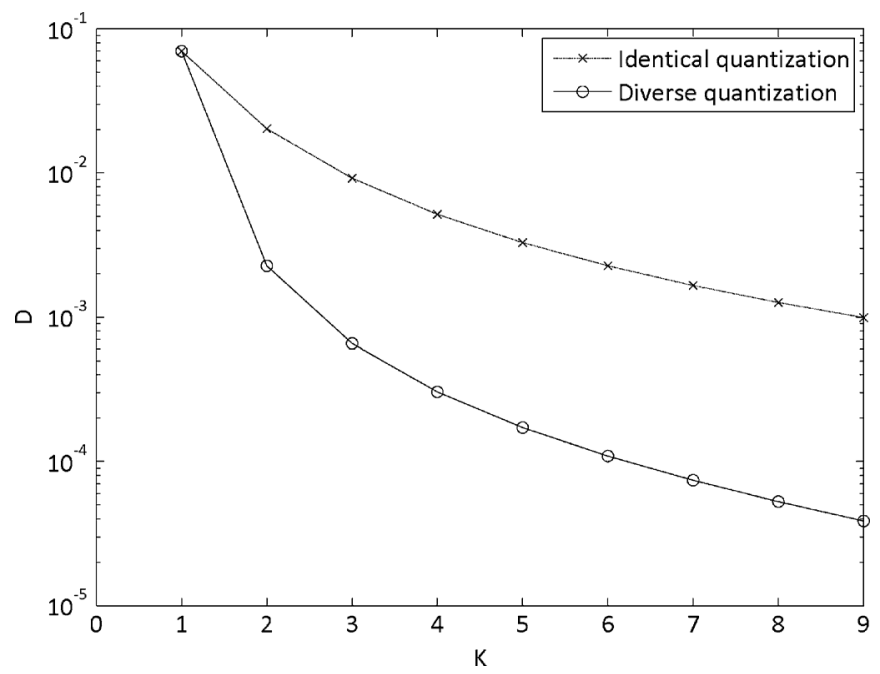

Fig. 8. Mean Bayes risk error for uniformly distributed $P_{0}$ in the example of Fig. 6.

\section{Exponential Likelihoods}

Consider a particle that disappears with rate $s_{0}$ in state $h_{0}$ and with rate $s_{1}$ in state $h_{1}$, where $s_{0}>s_{1}$. Conditioned on $H$, the particle has an exponentially distributed lifetime $Y$

$$
f_{Y^{\prime} H}\left(y \mid h_{m}\right)=s_{m} e^{-s_{m} y} .
$$

An agent observes that the particle disappears at time $Y=y$ and performs Bayesian hypothesis testing by the likelihood ratio test

$$
\frac{f_{Y \mid H}\left(y \mid h_{1}\right)}{f_{Y \mid H}\left(y \mid h_{0}\right)} \underset{\hat{H}=h_{0}}{\gtrless} \frac{p_{0} c_{10}}{\left(1-p_{0}\right) c_{01}} .
$$

The likelihood ratio test can be simplified to the decision rule

$$
y \underset{\hat{H}=h_{0}}{\gtrless} \frac{1}{s_{0}-s_{1}} \log \left(\frac{s_{0}}{s_{1}} \frac{p_{0} c_{10}}{\left(1-p_{0}\right) c_{01}}\right)=\lambda
$$

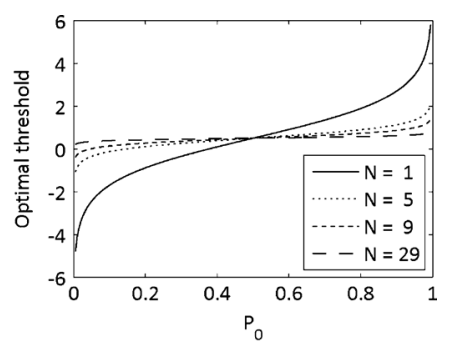

(a)

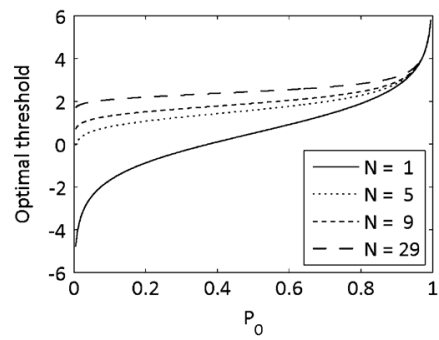

(b)

Fig. 9. Optimal decision threshold for $N$ agents for $c_{10}=c_{01}=1$ and $\sigma=1$. (a) MAJORITY rule. (b) OR rule.

which yields errors with probabilities

$$
P_{e}^{\mathrm{I}}=e^{-s_{0} \lambda} \quad \text { and } \quad P_{e}^{\mathrm{II}}=1-e^{-s_{1} \lambda} .
$$

Now suppose that $N$ agents perform Bayesian hypothesis testing with $N$ particles in the same state; Agent $i$ observes that the $i$ th particle disappears at time $Y_{i}=y_{i}$ and applies a common decision threshold $\lambda$ to its observation to make its local decision. All agents' decisions are fused by $L$-out-of- $N$ rule. Then the equivalent single-agent model is to consider a particle with lifetime $Y_{(L)}$, which is the $L$ th longest lifetime among $\left\{Y_{1}, \ldots, Y_{N}\right\}$

$$
\begin{aligned}
& f_{Y_{(L)} \mid H}\left(y \mid h_{m}\right) \\
& =\frac{N !}{(N-L) !(L-1) !}\left(1-e^{-s_{m} y}\right)^{N-L}\left(e^{-s_{m} y}\right)^{L-1} s_{m} e^{-s_{m} y} .
\end{aligned}
$$

The probabilities of global errors are

$$
\begin{aligned}
& P_{E}^{\mathrm{I}}=\sum_{n=L}^{N}\left(\begin{array}{l}
N \\
n
\end{array}\right)\left(e^{-s_{0} \lambda}\right)^{n}\left(1-e^{-s_{0} \lambda}\right)^{N-n}, \\
& P_{E}^{\mathrm{II}}=\sum_{n=N-L+1}^{N}\left(\begin{array}{l}
N \\
n
\end{array}\right)\left(1-e^{-s_{1} \lambda n}\right)^{n}\left(e^{-s_{1} \lambda}\right)^{N-n} .
\end{aligned}
$$

In this scenario, a small $L$ is a good choice for the fusion rule. Fig. 10(a) shows that the smallest mean Bayes risk without quantization of prior probabilities is achieved by $L=1$. Fig. 10(b) depicts an example of Bayes risk when five agents use diverse minimum MBRE quantizers and their fusion rule is the OR rule or the MAJORITY rule.

\section{Minimax BAyes Risk ERRor Quantizers}

Rather than mean Bayes risk error, let us consider maximum Bayes risk error as the criterion for optimizing quantizer design [8]. Such quantizers optimize worst case performance, whereas 


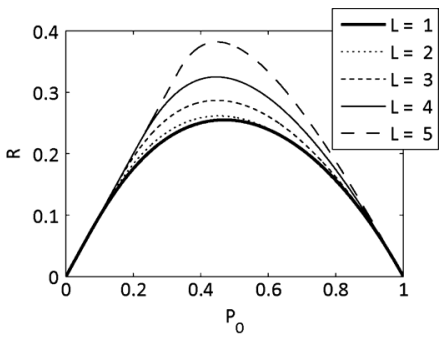

(a)

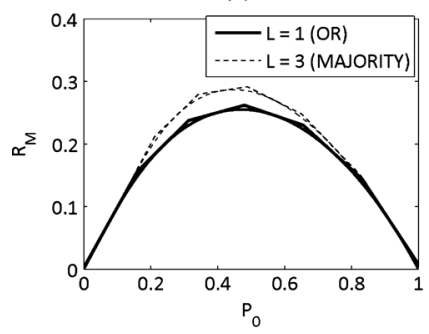

(b)

Fig. 10. Bayes risk (a) without quantization of prior probabilities and (b) with diverse 2-level minimum MBRE quantizers for $N=5, s_{0}=2, s_{1}=1$, $c_{10}=c_{01}=1$, and $u_{i}=1 / 5$ for $i=1, \ldots, 5$.

minimum MBRE quantizers optimize average performance. The minimax Bayes risk error quantizer is defined by the following optimization problem:

$$
\left(q_{1}^{*}, \ldots, q_{N}^{*}\right)=\underset{\left(q_{1}, \ldots, q_{N}\right)}{\arg \min } \max _{p_{0}} d\left(p_{0}, q_{1}\left(p_{0}\right), \ldots, q_{N}\left(p_{0}\right)\right) .
$$

The minimax Bayes risk error quantizer has the same nearest neighbor condition as the minimum MBRE quantizer for $N=$ 1. On the other hand, a centroid condition for optimality of a regular quantizer for $N=1$ is different from that of the minimum MBRE quantizer [8]. For any quantization point $a$, the Bayes risk error $d\left(p_{0}, a\right)=R_{M}(a)-R\left(p_{0}\right)$ is nonnegative and strictly convex in $p_{0}$, with minimum value of zero attained only at $p_{0}=a$. Thus, its maximum point within its $k$ th cell $\mathcal{R}_{k}=\left[b_{k-1}, b_{k}\right)$ is a cell boundary: $b_{k-1}$ or $b_{k}$. The point $a^{*}$ that satisfies

$$
d\left(b_{k-1}, a^{*}\right)=d\left(b_{k}, a^{*}\right)
$$

minimizes the maximum Bayes risk error within $\mathcal{R}_{k}$. This is the centroid condition for the representation point $a_{k}=a^{*}$ of cell $\mathcal{R}_{k}$. The minimax Bayes risk error quantizer can be found by alternatively applying the nearest neighbor condition (6) and the centroid condition (23) through the iterative Lloyd-Max algorithm.

For $N>1$, agents can take advantage of diversity in the same way as in Section IV-C. A team of $N$ agents bonded by the $L$-out-of- $N$ fusion rule is equivalent to a single agent with noise equal to their $L$ th largest noise $W_{(L)}$ (cf. Theorem 3). Thus, identical quantizers that minimize maximum Bayes risk error of the $N$ agents also minimize that of the single agent and vice versa (cf. Theorem 4).

Furthermore, if the $N$ agents collaborate by sharing the perceived common risk, there exists a set of $N$ diverse $K$-level regular quantizer that leads to the same Bayes risk for any $p_{0}$ as

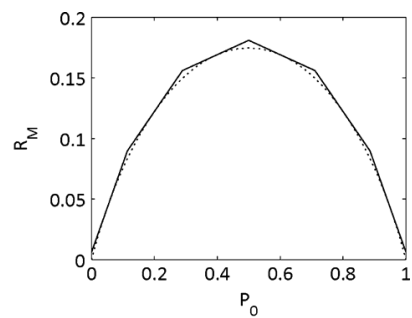

(a)

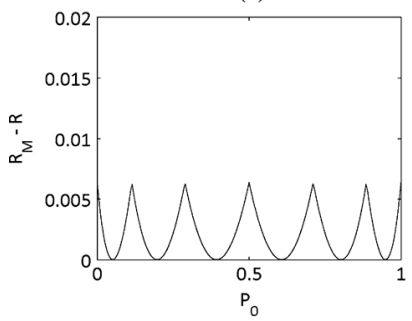

(b)

Fig. 11. (a) Bayes risk. (b) Bayes risk error for $N=5$ agents performing distributed hypothesis testing fused by the MAJORITY rule. They observe signals corrupted by i.i.d. additive Gaussian noise $\mathcal{N}\left(0, \sigma^{2}\right)$. The parameters are defined as $s_{0}=0, s_{1}=1, c_{10}=c_{01}=1, \sigma=1$, and $u_{i}=1 / 5$ for $i=1, \ldots, 5$.

a set of identical $(N(K-1)+1)$-level quantizers does (cf. Theorem 5). Therefore, the identical $(N(K-1)+1)$-level minimax Bayes risk error quantizers can be transformed into $N$ diverse $K$-level minimax Bayes risk error regular quantizers (cf. Theorem 6).

Fig. 11 shows an example of Bayes risk when five agents use diverse 2-level minimax Bayes risk error quantizers and their fusion rule is MAJORITY. A property of minimax Bayes risk error quantizers is that the maximum Bayes risk errors at all cell boundaries are the same, which is shown in Fig. 11(b). In addition, the minimax Bayes risk error quantizers are not dependent on the distribution of $P_{0}$ as long as $f_{P_{0}}\left(p_{0}\right)>0$ for all $p_{0} \in[0,1]$ because they minimize the worst case error, not the average.

\section{CONCLUSION}

We have discussed distributed detection and data fusion performed by a team of agents when there is a distribution of prior probabilities and the agents only know quantized versions of prior probabilities. When all agents use identical quantizers, they are affected by the same perceived Bayes risk and the distributed hypothesis testing problem can be analyzed by existing theorems of decision theory. On the contrary, when they do not use identical quantizers, then they consider different perceived Bayes risks, which prevents them from collaborating in hypothesis testing. We let the agents use the perceived common risk as a new distortion measure for hypothesis testing so as to unite them as a team to perform distributed hypothesis testing in any case.

We have defined mean Bayes risk error as the optimization criterion for prior-probability quantizers. We have presented theorems to show that diverse quantizers are better than identical quantizers. The equivalence between multiple-agent decision making and single-agent decision making simplifies 
a team of agents that use identical quantizers to a single agent. By combining the equivalence theorem with the equivalence between diverse quantizers and identical quantizers used by a team of agents, we can take advantage of the study of the minimum MBRE quantizer of a single agent to analyze optimal diverse regular quantization for multiple agents. It is shown that if the agents use diverse $K$-level regular quantizers, it has the same effect as using identical $(N(K-1)+1)$-level quantizers.

The assumption that each agent knows the others' quantization results and determines optimal identical decision rules leads to this strongly positive result. Without such an assumption, the agents are no longer able to collaborate to determine their decision rules. Each agent would optimize its decision rule based only on its own quantized value of prior probabilities, and consequently all agents will use suboptimal diverse decision rules. However, it is still better to use diverse quantizers for prior probabilities, as discussed in [27]-[29].

The equivalence theorems hold under the condition that all agents collaborate to perform Bayesian hypothesis testing. Hence, for any given distortion function of quantizers, diverse quantizers that minimize the distortion can be easily found. While the case of minimum MBRE was covered in detail, we also discussed the minimax Bayes risk error criterion. In the minimax case, one can again first design an $(N(K-1)+1)$-level quantizer for a single agent and then disassemble the quantizer into $N$ diverse $K$-level regular quantizers.

\section{REFERENCES}

[1] D. Austen-Smith and J. S. Banks, "Information aggregation, rationality, and the Condorcet jury theorem," Amer. Polit. Sci. Rev., vol. 90, no. 1, pp. 34-45, Mar. 1996.

[2] W. K. Viscusi, "Are individuals Bayesian decision makers?," Amer. Econ. Rev., vol. 75, no. 2, pp. 381-385, May 1985 .

[3] J. A. Swets, W. P. Tanner, Jr., and T. G. Birdsall, "Decision processes in perception," Psychol. Rev., vol. 68, no. 5, pp. 301-340, Sep. 1961.

[4] C. N. Macrae and G. V. Bodenhausen, "Social cognition: Thinking categorically about others," Ann. Rev. Psychol., vol. 51, pp. 93-120, Feb. 2000.

[5] K. J. R. Liu, H. Bourlard, V. Krishnamurthy, A. Pentland, and S. Wicker, "Introduction to the special issue on signal and information processing for social networks," IEEE J. Sel. Topics Signal Process., vol. 4, no. 4, pp. 673-676, Aug. 2010.

[6] K. R. Varshney, "Bayes risk error is a Bregman divergence," IEEE Trans. Signal Process., vol. 59, no. 9, pp. 4470-4472, Sep. 2011.

[7] K. R. Varshney and L. R. Varshney, "Quantization of prior probabilities for hypothesis testing," IEEE Trans. Signal Process., vol. 56, no. 10, pp. 4553-4562, Oct. 2008.

[8] K. R. Varshney and L. R. Varshney, "Multilevel minimax hypothesis testing," in Proc. IEEE/SP Workshop Stat. Signal Process., Nice, France, Jun. 2011, pp. 109-112.

[9] J. B. Rhim, L. R. Varshney, and V. K Goyal, "Collaboration in distributed hypothesis testing with quantized prior probabilities," in Proc. IEEE Data Compress. Conf., Snowbird, UT, Mar. 2011, pp. 303-312.

[10] J. Marschak and R. Radner, Economic Theory of Teams. New Haven, CT: Yale Univ. Press, 1972.

[11] J. B. Rhim, L. R. Varshney, and V. K Goyal, "Conflict in distributed hypothesis testing with quantized prior probabilities," in Proc. IEEE Data Compress. Conf., Snowbird, UT, Mar. 2011, pp. 313-322.

[12] S. Kassam, "Optimum quantization for signal detection," IEEE Trans. Commun., vol. COM-25, no. 5, pp. 479-484, May 1977.

[13] H. V. Poor and J. B. Thomas, "Applications of Ali-Silvey distance measures in the design of generalized quantizers for binary decision systems," IEEE Trans. Commun., vol. COM-25, no. 9, pp. 893-900, Sep. 1977.
[14] R. Gupta and A. O. Hero, III, "High-rate vector quantization for detection," IEEE Trans. Inf. Theory, vol. 49, no. 8, pp. 1951-1969, Aug. 2003.

[15] S. Kar and J. M. F. Moura, "Distributed consensus algorithms in sensor networks: Quantized data and random link failures," IEEE Trans. Signal Process., vol. 58, pp. 1383-1400, Mar. 2010.

[16] J. N. Tsitsiklis, "Decentralized detection," in Adv. Statist. Signal Process., H. V. Poor and J. B. Thomas, Eds. Greenwich, CT: JAI, 1993, pp. 297-344.

[17] R. Viswanathan and P. K. Varshney, "Distributed detection with multiple sensors: Part I-Fundamentals," Proc. IEEE, vol. 85, no. 1, pp. 54-63, Jan. 1997.

[18] G. A. Miller, "The magical number seven, plus or minus two: Some limits on our capacity for processing information," Psych. Rev., vol. 63, no. 2, pp. 81-97, Mar. 1956.

[19] R. Fryer and M. O. Jackson, "A categorical model of cognition and biased decision-making," B. E. J. Theor. Econ., vol. 8, no. 1, Jan. 2008.

[20] S. Mullainathan, J. Schwartzstein, and A. Shleifer, "Coarse thinking and persuasion," Quart. J. Econ., vol. 123, no. 2, pp. 577-619, May 2008.

[21] R. Radner, "Costly and bounded rationality in individual and team decision-making," in Understanding Industrial and Corporate Change, G. Dosi, D. J. Teece, and J. Chytry, Eds. Oxford, U.K.: Oxford Univ. Press, 2005, pp. 3-35.

[22] K. R. Varshney, "Frugal Hypothesis Testing and Classification," Ph.D. dissertation, Mass. Inst. Technol., Cambridge, Jun. 2010.

[23] M. Glanzer, A. Hilford, and L. T. Maloney, "Likelihood ratio decisions in memory: Three implied regularities," Psych. Bull. Rev., vol. 16, no. 3, pp. 431-455, Jun. 2009.

[24] G. L. Brase, L. Cosmides, and J. Tooby, "Individuation, counting, and statistical inference: The role of frequency and whole-object representations in judgment under uncertainty," J. Exp. Psychol. Gen., vol. 127, no. 1, pp. 3-21, Mar. 1998.

[25] J. N. Tsitsiklis, "Decentralized detection by a large number of sensors," Math. Contr., Signals, Syst., vol. 1, no. 2, pp. 167-182, 1988.

[26] H. A. David and H. N. Nagaraja, Order Statistics, 3rd ed. Hoboken, NJ: Wiley, 2003

[27] J. B. Rhim, L. R. Varshney, and V. K Goyal, "Distributed decision making by categorically-thinking agents," in Proc. NIPS 2011 Workshop on Decision Making With Multiple Imperfect Decision Makers, Sierra Nevada, Spain, Dec. 2011.

[28] J. B. Rhim, L. R. Varshney, and V. K Goyal, "Benefits of collaboration and diversity in teams of categorically-thinking decision makers," in Proc. IEEE Sens. Array and Multichannel Signal Process. Workshop, Hoboken, NJ, Jun. 2012, pp. 181-184.

[29] J. B. Rhim, L. R. Varshney, and V. K Goyal, "Distributed Decision Making by Categorically-Thinking Agents," in Decision Making With Imperfect Decision Makers. New York: Springer, to be published.

[30] L. H. C. Tippett, "On the extreme individuals and the range of samples taken from a normal population," Biometrica, vol. 17, no. 3/4, pp. 364-387, Dec. 1925.

[31] D. Teichroew, "Tables of expected values of order statistics and products of order statistics for samples of size twenty and less from the normal distribution," Ann. Math. Stat., vol. 27, no. 2, pp. 410-426, Jun. 1956.

[32] R. C. Bose and S. S. Gupta, "Moments of order statistics from a normal population," Biometrica, vol. 46, no. 3/4, pp. 433-440, Dec. 1959.

[33] L. R. Varshney, J. B. Rhim, K. R. Varshney, and V. K Goyal, "Categorical decision making by people, committees, and crowds," in Proc. Inf. Theory Appl. Workshop, La Jolla, CA, Feb. 2011.

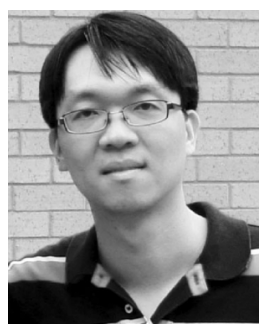

Joong Bum Rhim (S'11) received the B.S. degree with honors in electrical engineering (summa cum laude) from Korea Advanced Institute of Science and Technology (KAIST), Daejeon, in 2008. He received the S.M. degree in electrical engineering and computer science from the Massachusetts Institute of Technology (MIT), Cambridge, in 2010.

$\mathrm{He}$ is currently working toward the Ph.D. degree at MIT. He is a Research Assistant at MIT. His research interests include information theory, decision theory, machine learning, and game theory.

Mr. Rhim is a Korea Foundation for Advanced Studies Fellow. He was awarded the First Prize in the Student Paper Contest at the Seventh IEEE Sensor Array and Multichannel Signal Processing Workshop. 


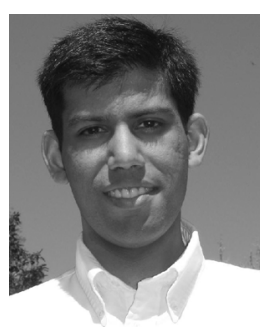

Lav R. Varshney (S'00-M'10) received the B.S degree with honors in electrical and computer engineering (magna cum laude) from Cornell University, Ithaca, NY, in 2004. He received the S.M., E.E., and $\mathrm{Ph} . \mathrm{D}$. degrees in electrical engineering and computer science from the Massachusetts Institute of Technology (MIT), Cambridge, in 2006, 2008, and 2010, respectively.

$\mathrm{He}$ is a research staff member with the IBM Thomas J. Watson Research Center, Hawthorne, NY. He has been a Postdoctoral Associate, National Science Foundation Graduate Research Fellow, and held various research and teaching positions at MIT. He has also held visiting positions at École Polytechnique Fédérale de Lausanne and at Cold Spring Harbor Laboratory. His current research interests include information theory, data analytics, sociotechnical systems, and neuroscience.

Dr. Varshney is a member of Tau Beta Pi, Eta Kappa Nu, and Sigma Xi. He received the J.-A. Kong Award Honorable Mention for Electrical Engineering doctoral thesis and the E. A. Guillemin Thesis Award for Outstanding Electrical Engineering S.M. Thesis.

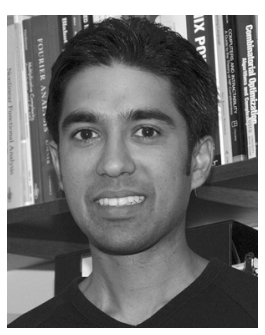

Vivek K Goyal (S'92-M'98-SM'03) received the B.S. degree in mathematics and the B.S.E. degree in electrical engineering from the University of Iowa, Iowa City, where he received the John Briggs Memorial Award for the top undergraduate across all colleges. He received the M.S. and Ph.D. degrees in electrical engineering from the University of California, Berkeley, where he received the Eliahu Jury Award for outstanding achievement in systems, communications, control, or signal processing.

He was a Member of Technical Staff in the Mathematics of Communications Research Department of Bell Laboratories, Lucent Technologies, 1998-2001; and a Senior Research Engineer for Digital Fountain, Inc., 2001-2003. He is currently Esther and Harold E. Edgerton Associate Professor of electrical engineering at the Massachusetts Institute of Technology. His research interests include computational imaging, sampling, quantization, and source coding theory.

Dr. Goyal is a member of Phi Beta Kappa, Tau Beta Pi, Sigma Xi, Eta Kappa $\mathrm{Nu}$, and SIAM. He was awarded the 2002 IEEE Signal Processing Society Magazine Award and an NSF CAREER Award. He served on the IEEE Signal Processing Society's Image and Multiple Dimensional Signal Processing Technical Committee. He is a Technical Program Committee Co-chair of IEEE ICIP 2016 and a permanent Conference Co-chair of the SPIE Wavelets and Sparsity conference series. 